\title{
Legendre-Gould Hopper based Sheffer polynomials: properties and applications
}

\author{
Ghazala Yasmin and Abdulghani Muhyi \\ Department of Applied Mathematics, \\ Faculty of Engineering and Technology \\ Aligarh Muslim University, Aligarh-202002, India.
}

\begin{abstract}
In this article, the Legendre-Gould Hopper polynomials are combined with Sheffer sequences to introduce certain mixed type special polynomials. Generating functions, differential equations and certain other properties of Legendre-Gould Hopper based Sheffer polynomials are derived. Further, operational and integral representations providing connections between these polynomials and known special polynomials are established. Certain identities and results for some members of these new mixed polynomials are also obtained. Finally, the determinantal definitions of Legendre-Gould Hopper based Sheffer polynomials are also given.
\end{abstract}

Keywords: Legendre-Gould Hopper based Sheffer polynomials; Generating relations; Operational method; Monomiality principle; Determinantal definition.

2010 Mathematics Subject Classification: 33C45, 33E20, 33C99.

\section{Introduction and preliminaries}

Operational methods developed within the context of the monomiality principle have opened new possibilities to treat problems associated with the properties of special functions and polynomials. Even classical problems with well known solutions may acquire a different flavor if viewed from such perspective. Furthermore, the same formalism provides the possibilities of introducing new families of mixed special polynomials. These polynomials have several applications in different branches of mathematics.

Recently, the Legendre-Gould Hopper polynomials (LeGHP) $\frac{{ }_{R} H_{n}^{(s)}(x, y, z)}{n !}$ and ${ }_{S} H_{n}^{(s)}(x, y, z)$ are introduced in [30] which are defined by means of the following generating functions

$$
\exp \left(z t^{s}\right) C_{0}(x t) C_{0}(-y t)=\sum_{n=0}^{\infty} \frac{{ }_{R} H_{n}^{(s)}(x, y, z)}{n !} \frac{t^{n}}{n !}
$$

and

$$
\exp \left(y t+z t^{s}\right) C_{0}\left(-x t^{2}\right)=\sum_{n=0}^{\infty}{ }_{S} H_{n}^{(s)}(x, y, z) \frac{t^{n}}{n !},
$$

E-mail: ghazala30@gmail.com (Ghazala Yasmin)

E-mail: muhyi2007@gmail.com (Abdulghani Muhyi) 
respectively, where $C_{0}(x)$ denotes the Tricomi function of order zero. The $n^{\text {th }}$ order Tricomi functions $C_{n}(x)$ are defined by means of the generating function

$$
\exp \left(t-\frac{x}{t}\right)=\sum_{n=0}^{\infty} C_{n}(x) t^{n}
$$

for $t \neq 0$ and for all finite $x$ and are defined by the following series [12, p.150]:

$$
C_{n}(x)=\sum_{s=0}^{\infty} \frac{(-1)^{s} x^{s}}{s !(n+s) !}, \quad n=0,1,2, \ldots, .
$$

The $0^{\text {th }}$-order Tricomi function $C_{0}(x)$ is also given by the following operational definition:

$$
C_{0}(\alpha x)=\exp \left(-\alpha D_{x}^{-1}\right)\{1\},
$$

where $D_{x}^{-1}$ denotes the inverse of the derivative operator $D_{x}:=\frac{\partial}{\partial x}$ and

$$
D_{x}^{-n}\{1\}=\frac{x^{n}}{n !}
$$

Also, it is known that $[16]$

$$
-\left(\frac{\partial}{\partial x} x \frac{\partial}{\partial x}\right) C_{0}(\alpha x)=\alpha C_{0}(\alpha x)
$$

The LeGHP $\frac{{ }_{R} H_{n}^{(s)}(x, y, z)}{n !}$ and ${ }_{S} H_{n}^{(s)}(x, y, z)$ are shown to be quasi-monomial $[12,28]$ under the action of the following multiplicative and derivative operators [30]:

$$
\begin{gathered}
\hat{M}_{R H}:=-D_{x}^{-1}+D_{y}^{-1}+s z \frac{\partial^{s-1}}{\partial y^{s-1}}, \\
\hat{P}_{R H}:=-\frac{\partial}{\partial x} x \frac{\partial}{\partial x}
\end{gathered}
$$

and

$$
\begin{gathered}
\hat{M}_{S H}:=y+2 D_{x}^{-1} \frac{\partial}{\partial y}+s z \frac{\partial^{s-1}}{\partial y^{s-1}}, \\
\hat{P}_{S H}:=\frac{\partial}{\partial y}
\end{gathered}
$$

respectively.

Consequently, $\hat{M}_{R H}, \hat{P}_{R H}$ and $\hat{M}_{S H}, \hat{P}_{S H}$ satisfy the following recurrence relations:

$$
\begin{aligned}
& \hat{M}_{R H}\left\{\frac{{ }_{R} H_{n}^{(s)}(x, y, z)}{n !}\right\}=\frac{{ }_{R} H_{n+1}^{(s)}(x, y, z)}{(n+1) !}, \\
& \hat{P}_{R H}\left\{\frac{{ }_{R} H_{n}^{(s)}(x, y, z)}{n !}\right\}=n \frac{{ }_{R} H_{n-1}^{(s)}(x, y, z)}{(n-1) !}
\end{aligned}
$$


and

$$
\begin{aligned}
& \hat{M}_{S H}\left\{{ }_{S} H_{n}^{(s)}(x, y, z)\right\}={ }_{S} H_{n+1}^{(s)}(x, y, z), \\
& \hat{P}_{S H}\left\{{ }_{S} H_{n}^{(s)}(x, y, z)\right\}=n_{S} H_{n-1}^{(s)}(x, y, z),
\end{aligned}
$$

respectively, for all $n \in \mathbb{N}$.

In view of the monomiality principle equations

$$
\begin{aligned}
& \hat{M}_{R H} \hat{P}_{R H}\left\{\frac{{ }_{R} H_{n}^{(s)}(x, y, z)}{n !}\right\}=n \frac{{ }_{R} H_{n}^{(s)}(x, y, z)}{n !}, \\
& \hat{M}_{S H} \hat{P}_{S H}\left\{{ }_{S} H_{n}^{(s)}(x, y, z)\right\}=n{ }_{S} H_{n}^{(s)}(x, y, z),
\end{aligned}
$$

the differential equations satisfied by $\frac{{ }_{R} H_{n}^{(s)}(x, y, z)}{n !}$ and ${ }_{S} H_{n}^{(s)}(x, y, z)$ are [30]:

$$
\left(-\frac{\partial}{\partial y}+s z \frac{\partial^{s+1}}{\partial x \partial y^{s}}+(1-n) \frac{\partial}{\partial x}\right){ }_{R} H_{n}^{(s)}(x, y, z)=0
$$

and

$$
\left(2 \frac{\partial^{2}}{\partial y^{2}}+s z \frac{\partial^{s+1}}{\partial x \partial y^{s}}+y \frac{\partial^{2}}{\partial x \partial y}-n \frac{\partial}{\partial x}\right){ }_{S} H_{n}^{(s)}(x, y, z)=0
$$

respectively.

Also, $\frac{{ }_{R} H_{n}^{(s)}(x, y, z)}{n !}$ and ${ }_{S} H_{n}^{(s)}(x, y, z)$ can be explicitly constructed as:

$$
\frac{{ }_{R} H_{n}^{(s)}(x, y, z)}{n !}=\hat{M}_{R H}^{n}\{1\}, \quad{ }_{R} H_{0}^{(s)}(x, y, z)=1
$$

and

$$
{ }_{S} H_{n}^{(s)}(x, y, z)=\hat{M}_{S H}^{n}\{1\}, \quad{ }_{S} H_{0}^{(s)}(x, y, z)=1,
$$

respectively.

Identities (1.21) and (1.20) imply that the exponential functions of $\frac{{ }_{R} H_{n}^{(s)}(x, y, z)}{n !}$ and ${ }_{S} H_{n}^{(s)}(x, y, z)$ can be given in the forms:

$$
\exp \left(t \hat{M}_{R H}\right)\{1\}=\sum_{n=0}^{\infty} \frac{{ }_{R} H_{n}^{(s)}(x, y, z)}{n !} \frac{t^{n}}{n !}, \quad|t|<\infty
$$

and

$$
\exp \left(t \hat{M}_{S H}\right)\{1\}=\sum_{n=0}^{\infty}{ }_{S} H_{n}^{(s)}(x, y, z) \frac{t^{n}}{n !}, \quad|t|<\infty
$$

respectively.

For suitable values of the indices and variables, the LeGHP $\frac{{ }_{R} H_{n}^{(s)}(x, y, z)}{n !}$ and ${ }_{S} H_{n}^{(s)}(x, y, z)$ give a number of other known special polynomials as special cases. We mention these special cases in Table 1. 
Table 1. List of special cases of $\operatorname{LeGHP}{ }_{S} H_{n}^{(s)}(x, y, z)$ and ${ }_{R} H_{n}^{(s)}(x, y, z)$

\begin{tabular}{|c|c|c|c|c|}
\hline \begin{tabular}{l|l} 
S. \\
No.
\end{tabular} & $\begin{array}{l}\text { Values of } \\
\text { the indices } \\
\text { and variables }\end{array}$ & $\begin{array}{l}\text { Relation between LeGHP } \\
{ }_{S} H_{n}^{(s)}(x, y, z),{ }_{R} H_{n}^{(s)}(x, y, z) \\
\text { and its special case }\end{array}$ & $\begin{array}{l}\text { Name of } \\
\text { known } \\
\text { polynomials }\end{array}$ & $\begin{array}{l}\text { Series definition } \\
\text { of known polynomials }\end{array}$ \\
\hline I. & $x=0$ & ${ }_{S} H_{n}^{(s)}(0, y, z)=H_{n}^{(s)}(y, z)$ & Gould-Hopper [22] & $H_{n}^{(s)}(x, y)=n ! \sum_{k=0}^{\left[\frac{n}{s}\right]} \frac{y^{k} x^{n-s k}}{k !(n-s k) !}$ \\
\hline II. & $z=0$ & ${ }_{S} H_{n}^{(s)}(x, y, 0)={ }_{2} L_{n}(x, y)$ & $\begin{array}{l}\text { 2-Variable Legendre } \\
\text { type }[17]\end{array}$ & ${ }_{2} L_{n}(x, y)=n ! \sum_{r=0}^{\left[\frac{n}{2}\right]} \frac{x^{r} y^{n-2 r}}{(r !)^{2}(n-2 r) !}$ \\
\hline III. & $\begin{array}{l}\text { i. } s=m ; x=0 \\
y \rightarrow-D_{x}^{-1}, z \rightarrow y \\
\text { ii. } s=m ; y=0, z \rightarrow y\end{array}$ & $\begin{array}{l}{ }_{S} H_{n}^{(m)}\left(0,-D_{x}^{-1}, y\right)={ }_{[m]} L_{n}(x, y) \\
{ }_{R} H_{n}^{(m)}(x, 0, y)={ }_{[m]} L_{n}(x, y)\end{array}$ & $\begin{array}{l}\text { 2-Variable } \\
\text { Generalized } \\
\text { Laguerre Type [14] }\end{array}$ & {$[m] L_{n}(x, y)=n ! \sum_{k=0}^{\left[\frac{n}{m}\right]} \frac{y^{k}(-x)^{n-m k}}{k ![(n-m k) !]^{2}}$} \\
\hline IV. & $\begin{array}{l}s=m-1 ; x=0 \\
y \rightarrow x, z \rightarrow y\end{array}$ & ${ }_{S} H_{n}^{(m-1)}(0, x, y)=U_{n}^{(m)}(x, y)$ & $\begin{array}{l}\text { Generalized } \\
\text { Chebyshev [13] }\end{array}$ & $U_{n}^{(m)}(x, y)=\sum_{k=0}^{\left[\frac{n}{m}\right]} \frac{(n-k) ! y^{k} x^{n-m k}}{k !(n-m k) !}$ \\
\hline V. & $\begin{array}{l}\text { i. } s=1, x=0, z \rightarrow-D_{x}^{-1} \\
\text { ii.s }=1 ; y=0, z \rightarrow y\end{array}$ & $\begin{array}{l}{ }_{S} H_{n}^{(1)}\left(0, y,-D_{x}^{-1}\right)=L_{n}(x, y) \\
{ }_{R} H_{n}^{(1)}(x, 0, y)=L_{n}(x, y)\end{array}$ & $\begin{array}{l}\text { 2-Variable } \\
\text { Laguerre [20] }\end{array}$ & $L_{n}(x, y)=n ! \sum_{r=0}^{n} \frac{(-x)^{r} y^{n-r}}{(r !)^{2}(n-r) !}$ \\
\hline VI. & $z=0$ & ${ }_{R} H_{n}^{(s)}(x, y, 0)=R_{n}(x, y)$ & $\begin{array}{l}\text { 2-Variable } \\
\text { Legendre [19] }\end{array}$ & $R_{n}(x, y)=(n !)^{2} \sum_{r=0}^{n} \frac{y^{r}(-x)^{n-r}}{(r !)^{2}[(n-r) !]^{2}}$ \\
\hline VII. & $\begin{array}{l}x=0, y \rightarrow x \\
z \rightarrow y D_{y} y\end{array}$ & ${ }_{S} H_{n}^{(s)}\left(0, x, y D_{y} y\right)=e_{n}^{(s)}(x, y)$ & $\begin{array}{l}\text { 2-Variable } \\
\text { truncated order of } \\
s[14]\end{array}$ & $e_{n}^{(s)}(x, y)=n ! \sum_{k=0}^{\left[\frac{n}{s}\right]} \frac{x^{n-s k} y^{k}}{(n-s k) !}$ \\
\hline VIII & $s=2 ; x=0$ & ${ }_{S} H_{n}^{(2)}(0, y, z)=H_{n}(y, z)$ & $\begin{array}{l}\text { 2-Variable } \\
\text { Hermite-Kampé } \\
\text { de Fériet }[2]\end{array}$ & $H_{n}(x, y)=n ! \sum_{k=0}^{\left[\frac{n}{2}\right]} \frac{y^{k} x^{n-2 k}}{k !(n-2 k)}$ \\
\hline IX. & $\begin{array}{l}\text { i. } s=2 ; x=0 \\
y \rightarrow D_{x}^{-1}, z \rightarrow y \\
\text { ii. } s=2 ; x=0 \\
y \rightarrow x, z \rightarrow y\end{array}$ & $\begin{array}{l}{ }_{S} H_{n}^{(2)}\left(0, D_{x}^{-1}, y\right)=G_{n}(x, y) \\
{ }_{R} H_{n}^{(2)}(0, x, y)=G_{n}(x, y)\end{array}$ & $\begin{array}{l}\text { Hermite } \\
\text { Type [15] }\end{array}$ & $G_{n}(x, y)=n ! \sum_{k=0}^{\left[\frac{n}{2}\right]} \frac{y^{k} x^{n-2 k}}{k ![(n-2 k) !]^{2}}$ \\
\hline $\mathrm{X}$. & $\begin{array}{l}\text { i. } x \rightarrow\left(\frac{x^{2}-1}{4}\right) \\
y \rightarrow x, z=0 \\
\text { ii.s }=1 ; x \rightarrow\left(\frac{1-x}{2}\right) \\
y \rightarrow\left(\frac{1+x}{2}\right), z=0\end{array}$ & $\begin{array}{l}{ }_{S} H_{n}^{(s)}\left(\frac{x^{2}-1}{4}, x, 0\right)=P_{n}(x) \\
{ }_{R} H_{n}^{(1)}\left(\frac{1-x}{2}, \frac{1+x}{2}, 0\right)=P_{n}(x)\end{array}$ & Legendre [26] & $P_{n}(x)=n ! \sum_{r=0}^{\left[\frac{n}{2}\right]} \frac{\left(x^{2}-1\right)^{k} x^{n-2 k}}{2^{2 k}(k !)^{2}(n-2 k) !}$ \\
\hline XI. & $\begin{array}{l}s=3 ;, x \rightarrow z D_{z} z \\
y \rightarrow x, z \rightarrow y\end{array}$ & ${ }_{S} H_{n}^{(3)}\left(z D_{z} z, x, y\right)=H_{n}^{(3,2)}(x, y, z)$ & Bell-Type [21] & $\begin{array}{l}H_{n}^{(3,2)}(x, y, z)=n ! \sum_{k=0}^{\left[\frac{n}{3}\right]} \frac{y^{k} H_{n-3 k}^{(2)}(x, z)}{k !(n-3 k) !} \\
=n ! \sum_{k=0}^{\left[\frac{n}{3}\right]} \sum_{r=0}^{\left[\frac{n-3 k}{2}\right]} \frac{y^{k} z^{r} x^{n-3 k-2 r}}{k ! r !(n-3 k-2 r) !}\end{array}$ \\
\hline
\end{tabular}

Next, we recall that the polynomial sequence $\left\{\mathbf{s}_{n}(x)\right\}_{n=0}^{\infty}\left(\mathbf{s}_{n}(x)\right.$ being a polynomial of degree $n$ ) is called Sheffer A-type zero [26, p.222 (Theorem 72)], (which we shall hereafter call Sheffer-type), if $\mathbf{s}_{n}(x)$ possesses the exponential generating function of the form

$$
A(t) \exp (x H(t))=\sum_{n=0}^{\infty} \mathbf{s}_{n}(x) \frac{t^{n}}{n !},
$$

where $A(t)$ and $H(t)$ have (at least the formal) expansions:

$$
A(t)=\sum_{n=0}^{\infty} A_{n} \frac{t^{n}}{n !}, \quad A_{0} \neq 0
$$

and

$$
H(t)=\sum_{n=1}^{\infty} H_{n} \frac{t^{n}}{n !}, \quad H_{1} \neq 0
$$

respectively.

Properties of Appell and Sheffer sequences are naturally handled within the framework of modern classical umbral calculus by Roman [27]. In view of the following result [27, p.17], the Sheffer sequences can be alternatively defined as: 
Let $f(t)$ be a delta series and $g(t)$ be an invertible series of the following form:

$$
f(t)=\sum_{n=0}^{\infty} f_{n} \frac{t^{n}}{n !}, \quad f_{0}=0, \quad f_{1} \neq 0
$$

and

$$
g(t)=\sum_{n=0}^{\infty} g_{n} \frac{t^{n}}{n !}, \quad g_{0} \neq 0 .
$$

Then there exists a unique sequence $\mathbf{s}_{n}(x)$ of polynomials satisfying the orthogonality conditions

$$
\left\langle g(t) f(t)^{k} \mid \mathbf{s}_{n}(x)\right\rangle=n ! \delta_{n, k}, \quad \text { for all } n, k \geq 0 \text {. }
$$

Also, in view of Roman [27, p.18 (Theorem 2.3.4)], the polynomial sequence $\mathbf{s}_{n}(x)$ is uniquely determined by two (formal) power series given by equations (1.27) and (1.28). The exponential generating function of $\mathbf{s}_{n}(x)$ is then given by

$$
\frac{1}{g\left(f^{-1}(t)\right)} \exp \left(x f^{-1}(t)\right)=\sum_{n=0}^{\infty} \mathbf{s}_{n}(x) \frac{t^{n}}{n !},
$$

for all $x \in \mathbb{C}$, where $f^{-1}(t)$ is the compositional inverse of $f(t)$. In view of equations (1.24) and (1.30), we have

$$
A(t)=\frac{1}{g\left(f^{-1}(t)\right)}
$$

and

$$
H(t)=f^{-1}(t)
$$

The sequence $\mathbf{s}_{n}(x)$ in equation (1.29) is the Sheffer sequence for the pair $(g(t), f(t))$. The Sheffer sequence for $(1, f(t))$ is called the associated Sheffer sequence for $f(t)$ and the Sheffer sequence for $(g(t), t)$ becomes the Appell sequence for $g(t)$ [27, p.17].

The Sheffer class contains very important sequences such as the Hermite, Laguerre,

\begin{tabular}{|c|c|c|c|c|c|}
\hline $\begin{array}{l}\text { S. } \\
\text { No. }\end{array}$ & $g(t) ; A(t)$ & $f(t) ; H(t)$ & Generating functions & $\hat{M}(X, D)$ and $\hat{P}(D)$ & Polynomials \\
\hline I. & $e^{\left(\frac{t}{v}\right)^{r}} ; e^{-t^{r}}$ & $\frac{t}{v} ; v t$ & $\begin{array}{l}\exp \left(v x t-t^{r}\right)= \\
\sum_{n=0}^{\infty} H_{n, r, v}(x) \frac{t^{n}}{n !}\end{array}$ & $\begin{array}{l}\hat{M}=v X-\frac{r}{v^{r-1}} D^{r-1} \\
\hat{P}=\frac{D}{v}\end{array}$ & $\begin{array}{l}\text { Generalized } \\
\text { Hermite } \\
\text { Polynomials } \\
H_{n, r, v}(x)[25] \\
\end{array}$ \\
\hline II. & $\begin{array}{l}(1-t)^{-\nu-1} \\
(1-t)^{-\nu-1}\end{array}$ & $\frac{t}{t-1} ; \frac{t}{t-1}$ & $\begin{array}{l}\frac{1}{(1-t) \nu+1} \exp \left(\frac{x t}{t-1}\right)= \\
\sum_{n=0}^{\infty} L_{n}^{(\nu)}(x) t^{n}\end{array}$ & $\begin{array}{l}\hat{M}=-X D^{2}+(2 X- \\
\nu-1) D-X+\nu+1 \\
\hat{P}=\frac{D}{D-1}\end{array}$ & $\begin{array}{l}\text { Generalized } \\
\text { Laguerre } \\
\text { Polynomials } \\
n ! L_{n}^{(\nu)}(x)[1,26]\end{array}$ \\
\hline III. & $\begin{array}{l}\frac{2}{e^{t}-1} ; \frac{t}{1-t} \\
(1-t)^{-\nu-1}\end{array}$ & $\frac{e^{t}-1}{e^{t}+1} ; \ln \left(\frac{1+t}{1-t}\right)$ & $\begin{array}{l}\frac{t}{1-t}\left(\frac{1+t}{1-t}\right)^{x}= \\
\sum_{n=0}^{\infty} P_{n}(x) t^{n}\end{array}$ & $\begin{array}{l}\hat{M}=\frac{X\left(1+e^{D}\right)^{2}}{2 e^{D}}-\frac{\left(1+e^{D}\right)^{2}}{2\left(1-e^{D}\right)} \\
\hat{P}=\frac{e^{D}-1}{e^{D}+1}\end{array}$ & $\begin{array}{l}\text { Pidduck } \\
\text { Polynomials } \\
P_{n}(x)[8,4] \\
\end{array}$ \\
\hline
\end{tabular}
Bernoulli, Poisson-Charlier polynomials etc. These polynomials are important from the view point of applications in physics, number theory and in many other branches of mathematics. We present the lists of some known Sheffer and associated Sheffer families in Tables 2 and 3 respectively.

Table 2. Some known Sheffer polynomials 


\begin{tabular}{|c|c|c|c|c|c|}
\hline IV. & $(1-t)^{-\beta} ; e^{\beta t}$ & $\ln (1-t) ; 1-e^{t}$ & $\begin{array}{l}\exp \left(\beta t+x\left(1-e^{t}\right)\right)= \\
\sum_{n=0}^{\infty} a_{n}^{(\beta)}(x) t^{n}\end{array}$ & $\begin{array}{l}\hat{M}=X D-X+\beta, \\
\hat{P}=\ln (1-D)\end{array}$ & $\begin{array}{l}\text { Acturial } \\
\text { Polynomials } \\
a_{n}^{(\beta)}(x)[8] \\
\end{array}$ \\
\hline V. & $\begin{array}{l}\exp \left(a\left(e^{t}-1\right)\right) \\
e^{-t}\end{array}$ & $\begin{array}{l}a\left(e^{t}-1\right) \\
\ln \left(1+\frac{t}{a}\right)\end{array}$ & $\begin{array}{l}e^{-t}\left(1+\frac{t}{a}\right)= \\
\sum_{n=0}^{\infty} c_{n}(x ; a) \frac{t^{n}}{n !}\end{array}$ & $\begin{array}{l}\hat{M}=\frac{X}{a e^{D}}-1, \\
\hat{P}=a\left(e^{D}-1\right)\end{array}$ & $\begin{array}{l}\text { Poisson-Charlier } \\
\text { Polynomials } \\
c_{n}(x ; a)[5,23] \\
\end{array}$ \\
\hline VI. & $\begin{array}{l}\left(1+e^{\lambda t}\right)^{\mu} \\
\left(1+(1+t)^{\lambda}\right)^{-\mu}\end{array}$ & $\begin{array}{l}e^{t}-1 \\
\ln (1+t)\end{array}$ & $\begin{array}{l}\left(1+(1+t)^{\lambda}\right)^{-\mu}(1+t)^{x}= \\
\sum_{n=0}^{\infty} s_{n}(x ; \lambda, \mu) \frac{t^{n}}{n !}\end{array}$ & $\begin{array}{l}\hat{M}=X e^{-D}-\frac{\mu \lambda e^{(\lambda-1) D}}{1+e^{\lambda D}} \\
\hat{P}=e^{D}-1\end{array}$ & $\begin{array}{l}\text { Peters } \\
\text { Polynomials } \\
s_{n}(x ; \lambda, \mu)[8] \\
\end{array}$ \\
\hline VII. & $\frac{t}{e^{t}-1} ; \frac{t}{\ln (1+t)}$ & $\begin{array}{l}e^{t}-1 \\
\ln (1+t)\end{array}$ & $\begin{array}{l}\frac{t}{\ln (1+t)}(1+t)^{x}= \\
\sum_{n=0}^{\infty} b_{n}(x) \frac{t^{n}}{n !}\end{array}$ & $\begin{array}{l}\hat{M}=X e^{-D}+\frac{D+e^{-D}-1}{D\left(e^{D}-1\right)} \\
\hat{P}=e^{D}-1\end{array}$ & $\begin{array}{l}\text { Bernoulli } \\
\text { Polynomials of } \\
\text { the second kind } \\
b_{n}(x)[23]\end{array}$ \\
\hline VIII & $\frac{1}{2}\left(1+e^{t}\right) ; \frac{2}{2+t}$ & $\begin{array}{l}e^{t}-1 \\
\ln (1+t)\end{array}$ & $\begin{array}{l}\frac{2}{2+t}(1+t)^{x}= \\
\sum_{n=0}^{\infty} r_{n}(x) \frac{t^{n}}{n !}\end{array}$ & $\begin{array}{l}\hat{M}=X e^{-D}-\frac{1}{\left(e^{D}+1\right)} \\
\hat{P}=e^{D}-1\end{array}$ & $\begin{array}{l}\text { Related } \\
\text { Polynomials } \\
r_{n}(x)[23]\end{array}$ \\
\hline IX. & sect $; \frac{1}{\sqrt{1+t^{2}}}$ & $\begin{array}{l}\operatorname{tant} ; \arctan (t) \\
\ln (1+t)\end{array}$ & $\begin{array}{l}\frac{1}{\sqrt{1+t^{2}}} \exp (\operatorname{xarctan}(t))= \\
\sum_{n=0}^{\infty} R_{n}(x) \frac{t^{n}}{n !}\end{array}$ & $\begin{array}{l}\hat{M}=(X-\tan D) \cos ^{2} D, \\
\hat{P}=\tan D\end{array}$ & $\begin{array}{l}\text { Hahn } \\
\text { Polynomials } \\
R_{n}(x)[7] \\
\end{array}$ \\
\hline $\mathrm{x}$. & $\begin{array}{l}\frac{1+t}{(1-t)^{a}} ; \\
(1-4 t)^{\frac{-1}{2}}\left(\frac{2}{1+\sqrt{1-4 t}}\right)^{a-1}\end{array}$ & $\begin{array}{l}\frac{1}{4}-\frac{1}{4}\left(\frac{1+t}{1-t}\right)^{2} \\
\frac{-4 t}{(1+\sqrt{1-4 t})^{2}}\end{array}$ & $\begin{array}{l}(1-4 t)^{\frac{-1}{2}}\left(\frac{2}{1+\sqrt{1-4 t}}\right)^{a-1} \\
\times \exp \left(\frac{-4 x t}{(1+\sqrt{1-4 t})^{2}}\right) \\
\sum_{n=0}^{\infty} R_{n}(a, x) t^{n}\end{array}$ & $\begin{array}{l}\hat{M}=\frac{-X(1-D)^{3}}{(1+D)}+ \\
\frac{(1-D)^{3}}{(1+D)^{2}}+\frac{a(1-D)^{3}}{(1+D)}, \\
\hat{P}=\frac{1}{4}-\frac{1}{4}\left(\frac{1+D}{1-D}\right)^{2}\end{array}$ & $\begin{array}{l}\text { Shively } \\
\text { Pseudo-Laguerre } \\
\text { Polynomials } \\
R_{n}(a, x)[26]\end{array}$ \\
\hline
\end{tabular}

Table 3. Some known associated Sheffer polynomials

\begin{tabular}{|l|l|l|l|l|}
\hline $\begin{array}{l}\text { S. } \\
\text { No. }\end{array}$ & $f(t) ; H(t)$ & Generating functions & $\hat{M}(X, D)$ and $\hat{P}(D)$ & Polynomials \\
\hline I. & $\frac{e^{t}-1}{e^{t}+t} ; \ln \left(\frac{1+t}{1-t}\right)$ & $\left(\frac{e^{t}-1}{e^{t}+t}\right)^{x}=\sum_{n=0}^{\infty} M_{n}(x) \frac{t^{n}}{n !}$ & $\begin{array}{l}\hat{M}=\frac{X\left(1+e^{D}\right)^{2}}{2 e^{D}}, \\
\hat{P}=\frac{e^{D}-1}{e^{D}+1}\end{array}$ & $\begin{array}{l}\text { Mittag-Leffler } \\
\text { polynomials } M_{n}(x)[3]\end{array}$ \\
\hline II. & $\ln (1+t) ; e^{t}-1$ & $\exp \left(x\left(e^{t}-1\right)\right)=\sum_{n=0}^{\infty} \phi_{n}(x) \frac{t^{n}}{n !}$ & $\begin{array}{l}\hat{M}=X(1+D), \\
\hat{P}=\ln (1+D)\end{array}$ & $\begin{array}{l}\text { Exponential } \\
\text { polynomials } \phi_{n}(x)[6]\end{array}$ \\
\hline III. & $\ln (1+t) ; e^{t}-1$ & $(1+t)^{x}=\sum_{n=0}^{\infty}(x)_{n} \frac{t^{n}}{n !}$ & $\begin{array}{l}\hat{M}=X e^{-D}, \\
\hat{P}=e^{D}-1\end{array}$ & $\begin{array}{l}\text { Lower factorial } \\
\text { polynomials }(x)_{n}[27]\end{array}$ \\
\hline IV. & $t-\frac{1}{2} t^{2} ; 1-\sqrt{1-2 t}$ & $\exp (x(1-\sqrt{1-2 t}))=\sum_{n=0}^{\infty} p_{n}(x) \frac{t^{n}}{n !}$ & $\begin{array}{l}\hat{M}=\frac{X}{1-D}, \\
\hat{P}=-t \frac{1}{2} D^{2}+D\end{array}$ & $\begin{array}{l}\text { Bessel polynomials } \\
p_{n}(x)[9,24]\end{array}$ \\
\hline
\end{tabular}

In the present paper, the Legendre-Gould Hopper based Sheffer polynomials are introduced and framed within the context of monomiality principle. Some operational and integral formulas for these polynomials are derived. Further, some results are obtained for some members of the Legendre-Gould Hopper based Sheffer polynomial families. The paper is also concluded with the determinantal definitions of the Legendre-Gould Hopper based Sheffer polynomials(LeGHSP) ${ }_{R} H^{(s)} \mathbf{S}_{n}(x, y, z)$ and ${ }_{S} H^{(s)} \mathbf{S}_{n}(x, y, z)$.

\section{Legendre-Gould Hopper based Sheffer polynomials}

To generate the Legendre-Gould Hopper based Sheffer polynomials (LeGHSP) denoted by ${ }_{R} H^{(s)} \mathbf{S}_{n}(x, y, z)$ and ${ }_{S} H^{(s)} \mathbf{s}_{n}(x, y, z)$, we prove the following results:

Theorem 2.1. The Legendre-Gould Hopper based Sheffer polynomials (LeGHSP) denoted by ${ }_{R} H^{(s)} \mathbf{S}_{n}(x, y, z)$ and ${ }_{S} H^{(s)} \mathbf{S}_{n}(x, y, z)$ are defined by the following generating functions

$$
\frac{1}{g\left(f^{-1}(t)\right)} \exp \left(z\left(f^{-1}(t)\right)^{s}\right) C_{0}\left(x f^{-1}(t)\right) C_{0}\left(-y f^{-1}(t)\right)=\sum_{n=0}^{\infty}{ }_{R} H^{(s)} \mathbf{s}_{n}(x, y, z) \frac{t^{n}}{n !}
$$


or, equivalently

$$
A(t) \exp \left(z(H(t))^{s}\right) C_{0}(x H(t)) C_{0}(-y H(t))=\sum_{n=0}^{\infty}{ }_{R} H^{(s)} \mathbf{s}_{n}(x, y, z) \frac{t^{n}}{n !}
$$

and

$$
\frac{1}{g\left(f^{-1}(t)\right)} \exp \left(y f^{-1}(t)+z\left(f^{-1}(t)\right)^{s}\right) C_{0}\left(-x\left(f^{-1}(t)\right)^{2}\right)=\sum_{n=0}^{\infty}{ }_{S} H^{(s)} \mathbf{s}_{n}(x, y, z) \frac{t^{n}}{n !},
$$

or, equivalently

$$
A(t) \exp \left(y H(t)+z(H(t))^{s}\right) C_{0}\left(-x(H(t))^{2}\right)=\sum_{n=0}^{\infty}{ }_{S} H^{(s)} \mathbf{s}_{n}(x, y, z) \frac{t^{n}}{n !},
$$

respectively.

Proof. Replacing $x$ in the l.h.s. and r.h.s. of equation (1.30) by the multiplicative operator $\hat{M}_{R H}$ of the LeGHP $\frac{{ }_{R} H_{n}^{(s)}(x, y, z)}{n !}$, we have

$$
\frac{1}{g\left(f^{-1}(t)\right)} \exp \left(\hat{M}_{R H} f^{-1}(t)\right)=\sum_{n=0}^{\infty} \mathbf{s}_{n}\left(\hat{M}_{R H}\right) \frac{t^{n}}{n !} .
$$

Using the expression of $\hat{M}_{R H}$ given in equation (1.8) and then decoupling the exponential operator in the l.h.s. of the resultant equation by using the Crofton-type identity [18, p.12]

$$
f\left(y+m \lambda \frac{d^{m-1}}{d y^{m-1}}\right)\{1\}=\exp \left(\lambda \frac{d^{m}}{d x^{m}}\right)\{f(y)\}
$$

we get

$$
\frac{1}{g\left(f^{-1}(t)\right)} \exp \left(z \frac{\partial^{s}}{\partial y^{s}}\right) \exp \left(\left(D_{x}^{-1}+D_{y}^{-1}\right) f^{-1}(t)\right)=\sum_{n=0}^{\infty} \mathbf{s}_{n}\left(-D_{x}^{-1}+D_{y}^{-1}+s z \frac{\partial^{s-1}}{\partial y^{s-1}}\right) \frac{t^{n}}{n !},
$$

Now, using the Weyl identity [18]

$$
e^{\hat{A}+\hat{B}}=e^{\hat{A}} e^{\hat{B}} e^{-k / 2}, \quad([\hat{A}, \hat{B}]=k, k \in \mathbb{C}),
$$

we get

$\frac{1}{g\left(f^{-1}(t)\right)} \exp \left(z \frac{\partial^{s}}{\partial y^{s}}\right) \exp \left(-D_{x}^{-1} f^{-1}(t)\right) \exp \left(D_{y}^{-1} f^{-1}(t)\right)=\sum_{n=0}^{\infty} \mathbf{s}_{n}\left(-D_{x}^{-1}+D_{y}^{-1}+s z \frac{\partial^{s-1}}{\partial y^{s-1}}\right) \frac{t^{n}}{n !}$,

Now, expanding the first exponential in the 1.h.s. of equation (2.9) and using definition (1.5), we find

$\frac{1}{g\left(f^{-1}(t)\right)} \exp \left(z\left(f^{-1}(t)\right)^{s}\right) C_{0}\left(x f^{-1}(t)\right) C_{0}\left(-y f^{-1}(t)\right)=\sum_{n=0}^{\infty} \mathbf{s}_{n}\left(-D_{x}^{-1}+D_{y}^{-1}+s z \frac{\partial^{s-1}}{\partial y^{s-1}}\right) \frac{t^{n}}{n !}$, 
Finally, denoting the resultant LeGHSP in the r.h.s. by ${ }_{R} H^{(s)} \mathbf{s}_{n}(x, y, z)$, that is

$$
{ }_{R} H^{(s)} \mathbf{s}_{n}(x, y, z)=\mathbf{s}_{n}\left(\hat{M}_{R H}\right)=\mathbf{s}_{n}\left(-D_{x}^{-1}+D_{y}^{-1}+s z \frac{\partial^{s-1}}{\partial y^{s-1}}\right),
$$

we get assertion (2.1). Also, in view of equations (1.31) and (1.32), generating function (2.1) can be expressed equivalently as equation (2.2). Making use of (1.10) and using a similar argument as in the above proof of (2.1), we can establish the assertions (2.3) and (2.4).

Next, to show that the LeGHSP ${ }_{R} H^{(s)} \mathbf{s}_{n}(x, y, z)$ and ${ }_{S} H^{(s)} \mathbf{s}_{n}(x, y, z)$ satisfy the monomiality property, we prove the following result:

Theorem 2.2. The Legendre-Gould Hopper based Sheffer polynomials ${ }_{R} H^{(s)} \mathbf{s}_{n}(x, y, z)$ and ${ }_{S} H^{(s)} \mathbf{S}_{n}(x, y, z)$ are quasi-monomial with respect to the following multiplicative and derivative operators:

$$
\begin{gathered}
\hat{M}_{R H \mathbf{s}}:=\left(-D_{x}^{-1}+D_{y}^{-1}+s z D_{y}^{s-1}-\frac{g^{\prime}\left(D_{y} y D_{y}\right)}{g\left(D_{y} y D_{y}\right)}\right) \frac{1}{f^{\prime}\left(D_{y} y D_{y}\right)}, \\
\hat{M}_{S H \mathbf{s}}:=\left(y+2 D_{x}^{-1} D_{y}+s z D_{y}^{s-1}-\frac{g^{\prime}\left(D_{y}\right)}{g\left(D_{y}\right)}\right) \frac{1}{f^{\prime}\left(D_{y}\right)},
\end{gathered}
$$

or, equivalently

$$
\begin{gathered}
\hat{M}_{R H \mathbf{s}}:=\left(-D_{x}^{-1}+D_{y}^{-1}+s z D_{y}^{s-1}\right) H^{\prime}\left(H^{-1}\left(D_{y} y D_{y}\right)\right)+\frac{A^{\prime}\left(H^{-1}\left(D_{y} y D_{y}\right)\right)}{A\left(H^{-1}\left(D_{y} y D_{y}\right)\right)}, \\
\hat{M}_{S H \mathbf{s}}:=\left(y+2 D_{x}^{-1} D_{y}+s z D_{y}^{s-1}\right) H^{\prime}\left(H^{-1}\left(D_{y}\right)\right)+\frac{A^{\prime}\left(H^{-1}\left(D_{y}\right)\right)}{A\left(H^{-1}\left(D_{y}\right)\right)}
\end{gathered}
$$

and

$$
\begin{gathered}
\hat{P}_{R H \mathbf{s}}:=f\left(D_{y} y D_{y}\right), \\
\hat{P}_{S H \mathbf{s}}:=f\left(D_{y}\right),
\end{gathered}
$$

or, equivalently

$$
\begin{gathered}
\hat{P}_{R H \mathbf{s}}:=H^{-1}\left(D_{y} y D_{y}\right), \\
\hat{P}_{S H \mathbf{s}}:=H^{-1}\left(D_{y}\right),
\end{gathered}
$$

respectively.

Proof. Consider the following identity:

$$
\left(D_{y} y D_{y}\right)\left\{C_{0}\left(-y f^{-1}(t)\right)\right\}=f^{-1}(t) C_{0}\left(-y f^{-1}(t)\right) .
$$

Since $f^{-1}$ denotes the compositional inverse of the function $f$ and $f(t)$ has an expansion (1.27) in powers of $t$, therefore we have

$$
f\left(D_{y} y D_{y}\right)\left\{C_{0}\left(-y f^{-1}(t)\right)\right\}=t C_{0}\left(-y f^{-1}(t)\right) .
$$


Differentiating equation (2.5) partially with respect to $t$ and in view of relation (2.11), we find

$$
\left(\left(\hat{M}_{R H}-\frac{g^{\prime}\left(f^{-1}(t)\right)}{g\left(f^{-1}(t)\right)}\right) \frac{1}{f^{\prime}\left(f^{-1}(t)\right)}\right) \frac{1}{g\left(f^{-1}(t)\right)} \exp \left(\hat{M}_{R H} f^{-1}(t)\right)=\sum_{n=0}^{\infty}{ }_{R} H^{(s)} \mathbf{s}_{n+1}(x, y, z) \frac{t^{n}}{n !},
$$

which on using monomiality principle equation (1.22) and definition (1.1) with $t=f^{-1}(t)$ gives

$$
\begin{array}{r}
\left(\left(\hat{M}_{R H}-\frac{g^{\prime}\left(f^{-1}(t)\right)}{g\left(f^{-1}(t)\right)}\right) \frac{1}{f^{\prime}\left(f^{-1}(t)\right)}\right) \frac{1}{g\left(f^{-1}(t)\right)} \exp \left(z\left(f^{-1}(t)\right)^{s}\right) C_{0}\left(x f^{-1}(t)\right) \\
\times C_{0}\left(-y f^{-1}(t)\right)=\sum_{n=0}^{\infty}{ }_{R} H^{(s)} \mathbf{S}_{n+1}(x, y, z) \frac{t^{n}}{n !}
\end{array}
$$

Since $g(t)$ is an invertible series and $f(t)$ is a delta series of $t$ therefore $\frac{g^{\prime}\left(f^{-1}(t)\right)}{g\left(f^{-1}(t)\right)}$ and $\frac{1}{f^{\prime}\left(f^{-1}(t)\right)}$ possess power series expansions of $f^{-1}(t)$. Thus, in view of relation $(2.20)$, the above equation becomes

$$
\begin{array}{r}
\left(\left(\hat{M}_{R H}-\frac{g^{\prime}\left(D_{y} y D_{y}\right)}{g\left(D_{y} y D_{y}\right)}\right) \frac{1}{f^{\prime}\left(D_{y} y D_{y}\right)}\right)\left\{\frac{1}{g\left(f^{-1}(t)\right)} \exp \left(z\left(f^{-1}(t)\right)^{s}\right) C_{0}\left(x f^{-1}(t)\right)\right. \\
\left.\times C_{0}\left(-y f^{-1}(t)\right)\right\}=\sum_{n=0}^{\infty}{ }_{R} H^{(s)} \mathbf{s}_{n+1}(x, y, z) \frac{t^{n}}{n !}
\end{array}
$$

which on using generating function (2.1) becomes

$$
\left(\left(\hat{M}_{R H}-\frac{g^{\prime}\left(D_{y} y D_{y}\right)}{g\left(D_{y} y D_{y}\right)}\right) \frac{1}{f^{\prime}\left(D_{y} y D_{y}\right)}\right)\left\{\sum_{n=0}^{\infty}{ }_{R} H^{(s)} \mathbf{s}_{n}(x, y, z) \frac{t^{n}}{n !}\right\}=\sum_{n=0}^{\infty}{ }_{R} H^{(s)} \mathbf{s}_{n+1}(x, y, z) \frac{t^{n}}{n !} .
$$

Adjusting the summation in the l.h.s. of the above equation and then equating the coefficients of like powers of $t$, we find

$$
\left(\left(\hat{M}_{R H}-\frac{g^{\prime}\left(D_{y} y D_{y}\right)}{g\left(D_{y} y D_{y}\right)}\right) \frac{1}{f^{\prime}\left(D_{y} y D_{y}\right)}\right)\left\{{ }_{R} H^{(s)} \mathbf{s}_{n}(x, y, z)\right\}={ }_{R} H^{(s)} \mathbf{s}_{n+1}(x, y, z),
$$

which, in view of equation (1.12) shows that the multiplicative operator for ${ }_{R} H^{(s)} \mathbf{S}_{n}(x, y, z)$ is given as:

$$
\hat{M}_{R H \mathbf{s}}=\left(\hat{M}_{R H}-\frac{g^{\prime}\left(D_{y} y D_{y}\right)}{g\left(D_{y} y D_{y}\right)}\right) \frac{1}{f^{\prime}\left(D_{y} y D_{y}\right)} .
$$

Finally, using equation (1.8) in the r.h.s of above equation, we get assertion (2.12).

Next, consider the following identity

$$
D_{y}\left\{\exp \left(y f^{-1}(t)+z\left(f^{-1}(t)\right)^{s}\right)\right\}=f^{-1}(t) \exp \left(y f^{-1}(t)+z\left(f^{-1}(t)\right)^{s}\right),
$$


and by making use of (1.10) and using a similar argument as in the above proof of (2.12), we establish the assertion (2.13).

Again, in view of identity (2.21), we have

$$
\begin{aligned}
f\left(D_{y} y D_{y}\right)\left\{\frac{1}{g\left(f^{-1}(t)\right)} \exp \left(z\left(f^{-1}(t)\right)^{s}\right) C_{0}\left(x f^{-1}(t)\right) C_{0}\left(-y f^{-1}(t)\right)\right\} \\
=t \frac{1}{g\left(f^{-1}(t)\right)} \exp \left(z\left(f^{-1}(t)\right)^{s}\right) C_{0}\left(x f^{-1}(t)\right) C_{0}\left(-y f^{-1}(t)\right)
\end{aligned}
$$

which on using generating function (2.1) becomes

$$
f\left(D_{y} y D_{y}\right)\left\{\sum_{n=0}^{\infty}{ }_{R} H^{(s)} \mathbf{s}_{n}(x, y, z) \frac{t^{n}}{n !}\right\}=\sum_{n=1}^{\infty}{ }_{R} H^{(s)} \mathbf{s}_{n-1}(x, y, z) \frac{t^{n}}{(n-1) !} .
$$

Adjusting the summation in the l.h.s. of the above equation and then equating the coefficients of like powers of $t$, we get

$$
f\left(D_{y} y D_{y}\right)\left\{{ }_{R} H^{(s)} \mathbf{S}_{n}(x, y, z)\right\}=n_{R} H^{(s)} \mathbf{S}_{n-1}(x, y, z), \quad n \geq 1,
$$

which in view of equation (1.13) yields assertion (2.16). Similarly, we can obtain the assertion (2.17). Also, in view of relations (1.31) and (1.32), assertions (2.12), (2.13), (2.16) and (2.17) can be expressed equivalently as equations (2.14), (2.15), (2.18) and (2.19), respectively.

In view of equation (1.20) and (1.21) and using equations (2.12), (2.13), (2.14) and (2.15), we deduce the following consequence of Theorem 2.2.

Corollary 2.1. The Legendre-Gould Hopper based Sheffer polynomials ${ }_{R} H^{(s)} \mathbf{S}_{n}(x, y, z)$ and ${ }_{S} H^{(s)} \mathbf{S}_{n}(x, y, z)$ have the following explicit representations:

$$
\begin{aligned}
& { }_{R} H^{(s)} \mathbf{s}_{n}(x, y, z)=\hat{M}_{R H \mathbf{s}}\{1\}, \\
& { }_{S} H^{(s)} \mathbf{s}_{n}(x, y, z)=\hat{M}_{S H \mathbf{s}}\{1\}
\end{aligned}
$$

that is,

$$
\begin{gathered}
{ }_{R} H^{(s)} \mathbf{S}_{n}(x, y, z)=\left(\left(-D_{x}^{-1}+D_{y}^{-1}+s z D_{y}^{s-1}-\frac{g^{\prime}\left(D_{y} y D_{y}\right)}{g\left(D_{y} y D_{y}\right)}\right) \frac{1}{f^{\prime}\left(D_{y} y D_{y}\right)}\right)^{n}\{1\}, \\
{ }_{S} H^{(s)} \mathbf{s}_{n}(x, y, z)=\left(\left(y+2 D_{x}^{-1} D_{y}+s z D_{y}^{s-1}-\frac{g^{\prime}\left(D_{y}\right)}{g\left(D_{y}\right)}\right) \frac{1}{f^{\prime}\left(D_{y}\right)}\right)^{n}\{1\},
\end{gathered}
$$

or, equivalently

$$
\begin{array}{r}
{ }_{R} H^{(s)} \mathbf{S}_{n}(x, y, z)=\left(\left(-D_{x}^{-1}+D_{y}^{-1}+s z D_{y}^{s-1}\right) H^{\prime}\left(H^{-1}\left(D_{y} y D_{y}\right)\right)+\frac{A^{\prime}\left(H^{-1}\left(D_{y} y D_{y}\right)\right)}{A\left(H^{-1}\left(D_{y} y D_{y}\right)\right)}\right)^{n}\{1\}, \\
{ }_{S} H^{(s)} \mathbf{S}_{n}(x, y, z)=((y+2.36)
\end{array}
$$

respectively. 
Theorem 2.3. The Legendre-Gould Hopper based Sheffer polynomials ${ }_{R} H^{(s)} \mathbf{s}_{n}(x, y, z)$ and ${ }_{S} H^{(s)} \mathbf{S}_{n}(x, y, z)$ are the solutions of the following differential equations:

$$
\left(\left(-D_{x}^{-1}+D_{y}^{-1}+s z D_{y}^{s-1}-\frac{g^{\prime}\left(D_{y} y D_{y}\right)}{g\left(D_{y} y D_{y}\right)}\right) \frac{f\left(D_{y} y D_{y}\right)}{f^{\prime}\left(D_{y} y D_{y}\right)}-n\right){ }_{R} H^{(s)} \mathbf{s}_{n}(x, y, z)=0,
$$

or, equivalently

$$
\begin{aligned}
\left(\left(\left(-D_{x}^{-1}+D_{y}^{-1}+s z D_{y}^{s-1}\right) H^{\prime}\left(H^{-1}\left(D_{y} y D_{y}\right)\right)\right.\right. \\
\left.\left.\quad+\frac{A^{\prime}\left(H^{-1}\left(D_{y} y D_{y}\right)\right)}{A\left(H^{-1}\left(D_{y} y D_{y}\right)\right)}\right) H^{-1}\left(D_{y} y D_{y}\right)-n\right){ }_{R} H^{(s)} \mathbf{s}_{n}(x, y, z)=0
\end{aligned}
$$

and

$$
\left(\left(y+2 D_{x}^{-1} D_{y}+s z D_{y}^{s-1}-\frac{g^{\prime}\left(D_{y}\right)}{g\left(D_{y}\right)}\right) \frac{f\left(D_{y}\right)}{f^{\prime}\left(D_{y}\right)}-n\right)_{s H^{(s)}} \mathbf{s}_{n}(x, y, z)=0
$$

or, equivalently

$$
\left(\left(\left(y+2 D_{x}^{-1} D_{y}+s z D_{y}^{s-1}\right) H^{\prime}\left(H^{-1}\left(D_{y}\right)\right)+\frac{A^{\prime}\left(H^{-1}\left(D_{y}\right)\right)}{A\left(H^{-1}\left(D_{y}\right)\right)}\right) H^{-1}\left(D_{y}\right)-n\right){ }_{s} H^{(s)} \mathbf{s}_{n}(x, y, z)=0,
$$

respectively.

Proof. Using equations (2.12) and (2.16) in the corresponding equation (1.16) for the LegendreGould Hopper based Sheffer polynomials ${ }_{R} H^{(s)} \mathbf{S}_{n}(x, y, z)$, we get assertion (2.38). Also, using equations (2.14) and (2.15) in the corresponding equation (1.17) for the Legendre-Gould Hopper based Sheffer polynomials ${ }_{S} H^{(s)} \mathbf{S}_{n}(x, y, z)$, we get assertion (2.40). Using similar argument, we can get assertion (2.39) and assertion (2.41).

Remark 2.1. Since, for $g(t)=1$ (or $A(t)=1)$, the Sheffer polynomials $\mathbf{s}_{n}(x)$ reduce to the associated Sheffer polynomials $\mathfrak{s}_{n}(x)$. Therefore, taking $g(t)=1$ (or $A(t)=1$ ) in the results obtained in Theorems 2.1-2.3 and denoting the resultant Legendre-Gould Hopper based associated Sheffer polynomials (LeGHASP) by ${ }_{R} H^{(s)} \mathfrak{s}_{n}(x, y, z)$ and ${ }_{S} H^{(s)} \mathfrak{s}_{n}(x, y, z)$, we deduce the following consequences of Theorems 2.1-2.3:

Corollary 2.2. The Legendre-Gould Hopper based associated Sheffer polynomials (LeGHASP) denoted by ${ }_{R} H^{(s)} \mathfrak{s}_{n}(x, y, z)$ and ${ }_{S} H^{(s)} \mathfrak{s}_{n}(x, y, z)$ are defined by the following generating functions

$$
\exp \left(z\left(f^{-1}(t)\right)^{s}\right) C_{0}\left(x f^{-1}(t)\right) C_{0}\left(-y f^{-1}(t)\right)=\sum_{n=0}^{\infty}{ }_{R} H^{(s)} \mathfrak{s}_{n}(x, y, z) \frac{t^{n}}{n !},
$$

or, equivalently

$$
\exp \left(z(H(t))^{s}\right) C_{0}(x H(t)) C_{0}(-y H(t))=\sum_{n=0}^{\infty}{ }_{R} H^{(s)} \mathfrak{s}_{n}(x, y, z) \frac{t^{n}}{n !}
$$


and

$$
\exp \left(y f^{-1}(t)+z\left(f^{-1}(t)\right)^{s}\right) C_{0}\left(-x\left(f^{-1}(t)\right)^{2}\right)=\sum_{n=0}^{\infty}{ }_{S} H^{(s)} \mathfrak{s}_{n}(x, y, z) \frac{t^{n}}{n !}
$$

or, equivalently

$$
\exp \left(y H(t)+z(H(t))^{s}\right) C_{0}\left(-x(H(t))^{2}\right)=\sum_{n=0}^{\infty}{ }_{S} H^{(s)} \mathfrak{s}_{n}(x, y, z) \frac{t^{n}}{n !},
$$

respectively.

Corollary 2.3. The Legendre-Gould Hopper based associated Sheffer polynomials ${ }_{R} H^{(s)} \mathfrak{s}_{n}(x, y, z)$ and ${ }_{S} H^{(s)} \mathfrak{s}_{n}(x, y, z)$ are quasi-monomial with respect to the following multiplicative and derivative operators:

$$
\begin{gathered}
\hat{M}_{R H \mathfrak{s}}:=\left(-D_{x}^{-1}+D_{y}^{-1}+s z D_{y}^{s-1}\right) \frac{1}{f^{\prime}\left(D_{y} y D_{y}\right)}, \\
\hat{M}_{S H \mathfrak{s}}:=\left(y+2 D_{x}^{-1} D_{y}+s z D_{y}^{s-1}\right) \frac{1}{f^{\prime}\left(D_{y}\right)},
\end{gathered}
$$

or, equivalently

$$
\begin{gathered}
\hat{M}_{R H_{\mathfrak{s}}}:=\left(-D_{x}^{-1}+D_{y}^{-1}+s z D_{y}^{s-1}\right) H^{\prime}\left(H^{-1}\left(D_{y} y D_{y}\right)\right), \\
\hat{M}_{S H_{\mathfrak{s}}}:=\left(y+2 D_{x}^{-1} D_{y}+s z D_{y}^{s-1}\right) H^{\prime}\left(H^{-1}\left(D_{y}\right)\right)
\end{gathered}
$$

and

or, equivalently

$$
\begin{gathered}
\hat{P}_{R H_{\mathfrak{s}}}:=f\left(D_{y} y D_{y}\right), \\
\hat{P}_{S H_{\mathfrak{s}}}:=f\left(D_{y}\right),
\end{gathered}
$$

$$
\begin{gathered}
\hat{P}_{R H \mathfrak{s}}:=H^{-1}\left(D_{y} y D_{y}\right), \\
\hat{P}_{S H_{\mathfrak{s}}}:=H^{-1}\left(D_{y}\right),
\end{gathered}
$$

respectively.

Corollary 2.4. The Legendre-Gould Hopper based associated Sheffer polynomials ${ }_{R} H^{(s)} \mathfrak{s}_{n}(x, y, z)$ and ${ }_{S H^{(s)}} \mathfrak{s}_{n}(x, y, z)$ are the solutions of the following differential equations:

$$
\left(\left(-D_{x}^{-1}+D_{y}^{-1}+s z D_{y}^{s-1}\right) \frac{f\left(D_{y} y D_{y}\right)}{f^{\prime}\left(D_{y} y D_{y}\right)}-n\right)_{R} H^{(s)} \mathfrak{s}_{n}(x, y, z)=0,
$$

or, equivalently

$$
\left(\left(\left(-D_{x}^{-1}+D_{y}^{-1}+s z D_{y}^{s-1}\right) H^{\prime}\left(H^{-1}\left(D_{y} y D_{y}\right)\right)\right) H^{-1}\left(D_{y} y D_{y}\right)-n\right)_{R} H^{(s)} \mathfrak{s}_{n}(x, y, z)=0
$$

and

$$
\left(\left(y+2 D_{x}^{-1} D_{y}+s z D_{y}^{s-1}\right) \frac{f\left(D_{y}\right)}{f^{\prime}\left(D_{y}\right)}-n\right){ }_{s} H^{(s)} \mathfrak{s}_{n}(x, y, z)=0
$$

or, equivalently

$$
\left(\left(\left(y+2 D_{x}^{-1} D_{y}+s z D_{y}^{s-1}\right) H^{\prime}\left(H^{-1}\left(D_{y}\right)\right)\right) H^{-1}\left(D_{y}\right)-n\right)_{S} H^{(s)} \mathfrak{s}_{n}(x, y, z)=0,
$$

respectively. 
We have mentioned special cases of the $\operatorname{LeGHP}{ }_{S} H_{n}^{(s)}(x, y, z)$ and $\frac{{ }_{R} H_{n}^{(s)}(x, y, z)}{n !}$ in Table 1. Now, for the same choice of the variables and indices the $\operatorname{LeGHSP}_{R} H^{(s)} \mathbf{s}_{n}(x, y, z)$ and ${ }_{S} H^{(s)} \mathbf{S}_{n}(x, y, z)$ reduce to the corresponding special cases. We mention these known and new special polynomials related to the Sheffer sequences in Table 4.

Table 4. Special cases of LeGHSP ${ }_{R} H^{(s)} \mathbf{S}_{n}(x, y, z)$ and ${ }_{S} H^{(s)} \mathbf{s}_{n}(x, y, z)$

\begin{tabular}{|c|c|c|c|}
\hline $\begin{array}{l}\text { S. } \\
\text { No. }\end{array}$ & $\begin{array}{l}\text { Values of the indices } \\
\text { and variables }\end{array}$ & $\begin{array}{l}\text { Relation between LeGHSP }{ }_{R^{H}(s)} \mathbf{s}_{n}(x, y, z) \text {, } \\
{ }^{(s)} \mathbf{s}_{n}(x, y, z) \text { and its special case }\end{array}$ & $\begin{array}{l}\text { Name of the } \\
\text { special polynomials }\end{array}$ \\
\hline I. & $x=0$ & ${ }_{S} H^{(s)} \mathbf{s}_{n}(0, y, z)={ }_{H}(s) \mathbf{s}_{n}(y, z)$ & $\begin{array}{l}\text { Gould-Hopper based } \\
\text { Sheffer polynomials (GHSP) }\end{array}$ \\
\hline II. & $z=0$ & ${ }_{S}{ }^{H(s)} \mathbf{s}_{n}(x, y, 0)={ }_{2}{ }^{L} \mathbf{s}_{n}(x, y)$ & $\begin{array}{l}\text { 2-Variable Legendre based } \\
\text { Sheffer polynomials (2VLeSP) }\end{array}$ \\
\hline III. & $\begin{array}{l}\text { i. } s=m ; x=0 \\
y \rightarrow-D_{x}^{-1}, z \rightarrow y \\
\text { ii.s }=m ; y=0, z \rightarrow y\end{array}$ & $\begin{array}{l}{ }_{S} H^{(m)} \mathbf{s}_{n}\left(\frac{-x}{t}, 0, y\right)={ }_{[m]}{ }^{L} \mathbf{s}_{n}(x, y) \\
{ }_{R} H^{(m)} \mathbf{s}_{n}(x, 0, y)={ }_{[m]} \mathbf{s}_{n}(x, y)\end{array}$ & $\begin{array}{l}\text { 2-Variable Generalized } \\
\text { Laguerre Type based Sheffer } \\
\text { polynomials (2VGLTSP) }\end{array}$ \\
\hline IV. & $\begin{array}{l}s=m-1 ; x=0 \\
y \rightarrow x, z \rightarrow y\end{array}$ & ${ }_{S} H^{(m-1)} \mathbf{s}_{n}(0, x, y)={ }_{U}(m) \mathbf{s}_{n}(x, y)$ & $\begin{array}{l}\text { Generalized Chebyshev based } \\
\text { Sheffer polynomials (GCSP) }\end{array}$ \\
\hline V. & $\begin{array}{l}\text { i. } s=1, x=0, z=0 \\
\text { ii. } s=1 ; y=0, z \rightarrow y\end{array}$ & $\begin{array}{l}{ }_{S} H^{(1)} \mathbf{s}_{n}\left(0, y,-D_{x}^{-1}\right)={ }_{L} \mathbf{s}_{n}(x, y) \\
{ }_{R}^{H}{ }^{(1)} \mathbf{s}_{n}(x, o, y)={ }_{L} \mathbf{s}_{n}(x, y)\end{array}$ & $\begin{array}{l}\text { 2-Variable Laguerre based } \\
\text { Sheffer polynomials (2VLSP) }\end{array}$ \\
\hline VI. & $z=0$ & $R^{H^{(s)} \mathbf{s}_{n}(x, y, 0)}=R_{\mathbf{s}_{n}(x, y)}$ & $\begin{array}{l}\text { 2-Variable Legendre based } \\
\text { Sheffer polynomials (2VLeSP) }\end{array}$ \\
\hline VII. & $\begin{array}{l}x=0, y \rightarrow x \\
z \rightarrow y D_{y} y\end{array}$ & ${ }_{S} H^{(s)} \mathbf{s}_{n}\left(0, x, y D_{y} y\right)=e^{(s)} \mathbf{s}_{n}(x, y)$ & $\begin{array}{l}\text { 2-Variable truncated } \\
\text { based Sheffer polynomials of } \\
\text { order } s \text { (2VTSP) }\end{array}$ \\
\hline VIII. & $s=2 ; x=0$ & ${ }_{S} H^{(2)} \mathbf{s}_{n}(0, y, z)={ }_{H} \mathbf{s}_{n}(y, z)$ & $\begin{array}{l}\text { 2-Variable Hermite-Kamp } \\
\text { de Fériet é based Sheffer } \\
\text { polynomials (2VHKFSP) }\end{array}$ \\
\hline IX. & $\begin{array}{l}\text { i. } s=2 ; x=0 \\
y \rightarrow D_{x}^{-1}, z \rightarrow y \\
\text { ii. } s=2 ; x=0 \\
y \rightarrow x, z \rightarrow y\end{array}$ & $\begin{array}{l}{ }_{S} H^{(2)} \mathbf{s}_{n}\left(0, D_{x}^{-1}, y\right)={ }_{G} \mathbf{s}_{n}(x, y) \\
{ }_{R} H^{(2)} \mathbf{s}_{n}(0, x, y)={ }_{G} \mathbf{s}_{n}(x, y)\end{array}$ & $\begin{array}{l}\text { Hermite Type based Sheffer } \\
\text { polynomials (HTSP) }\end{array}$ \\
\hline $\mathrm{X}$. & $\begin{array}{l}\text { i. } x \rightarrow\left(\frac{x^{2}-1}{4}\right) \\
y \rightarrow x, z=0 \\
\text { ii. } s=1 ; x \rightarrow\left(\frac{1-x}{2}\right) \\
y \rightarrow\left(\frac{1+x}{2}\right), z=0\end{array}$ & $\begin{array}{l}S^{(s)} \mathbf{s}_{n}\left(\frac{x^{2}-1}{4}, x, 0\right)={ }_{P} \mathbf{s}_{n}(x) \\
R^{H^{(1)}} \mathbf{s}_{n}\left(\frac{1-x}{2}, \frac{1+x}{2}, 0\right)={ }_{P} \mathbf{s}_{n}(x)\end{array}$ & $\begin{array}{l}\text { Legendre based Sheffer } \\
\text { polynomials (LeSP) }\end{array}$ \\
\hline XI. & $\begin{array}{l}s=3 ; x \rightarrow z D_{z} z \\
y \rightarrow x, z \rightarrow y\end{array}$ & ${ }_{S} H^{(3)} \mathbf{s}_{n}\left(z D_{z} z, x, y\right)={ }_{H}^{(3,2)} \mathbf{s}_{n}(x, y, z)$ & $\begin{array}{l}\text { Bell-Type based Sheffer } \\
\text { polynomials (BTSP) }\end{array}$ \\
\hline
\end{tabular}

Remark 2.2. In view of the special cases mentioned in Table 4, the results for the special polynomials related to the Sheffer sequences can be obtained.

Next, we derive certain operational representations for the LeGHSP ${ }_{R} H^{(s)} \mathbf{S}_{n}(x, y, z)$ and ${ }_{S} H^{(s)} \mathbf{s}_{n}(x, y, z)$.

\section{Operational and integral representations}

To establish the operational representation for the $\operatorname{LeGHSP}_{R} H^{(s)} \mathbf{s}_{n}(x, y, z)$ and ${ }_{S} H^{(s)} \mathbf{s}_{n}(x, y, z)$, we prove the following results:

Theorem 3.1. The following operational representation between the LeGHSP ${ }_{R} H^{(s)} \mathbf{S}_{n}(x, y, z)$, ${ }_{S} H^{(s)} \mathbf{s}_{n}(x, y, z)$ and the Sheffer polynomials $\mathbf{s}_{n}(x)$ hold true:

$$
{ }_{R} H^{(s)} \mathbf{s}_{n}(x, y, z)=\exp \left(z \frac{\partial^{s}}{\partial y^{s}}\right) \mathbf{s}_{n}\left(-D_{x}^{-1}+D_{y}^{-1}\right)
$$

and

$$
{ }_{S} H^{(s)} \mathbf{s}_{n}(x, y, z)=\exp \left(D_{x}^{-1} \frac{\partial^{2}}{\partial y^{2}}+z \frac{\partial^{s}}{\partial y^{s}}\right) \mathbf{s}_{n}(y)
$$


respectively.

Proof. In view of equation (2.11), the proof of (3.1) is direct use of identity (2.6) and similarly the proof of (3.2) can be obtained.

Theorem 3.2. The following operational representation between the LeGHSP ${ }_{R} H^{(s)} \mathbf{s}_{n}(x, y, z)$ and the $2 V L e S P{ }_{R} \mathbf{s}_{n}(x, y)$ holds true:

$$
{ }_{R} H^{(s)} \mathbf{S}_{n}(x, y, z)=\exp \left((-1)^{s} z \frac{\partial^{s}}{\partial D_{x}^{-s}}\right){ }_{R} \mathbf{s}_{n}(x, y),
$$

or, equivalently

$$
{ }_{R} H^{(s)} \mathbf{S}_{n}(x, y, z)=\exp \left(z \frac{\partial^{s}}{\partial D_{y}^{-s}}\right){ }_{R} \mathbf{s}_{n}(x, y) .
$$

Proof. From equation (2.2), we have

$$
(-1)^{s} \frac{\partial^{s}}{\partial D_{x}^{-s}}{ }_{R} H^{(s)} \mathbf{s}_{n}(x, y, z)=\frac{\partial}{\partial z}{ }_{R} H^{(s)} A_{n}(x, y, z) .
$$

Since, in view of Table $1(\mathrm{VI})$, we have

$$
{ }_{R} H_{n}^{(s)}(x, y, 0)=R_{n}(x, y)
$$

Consequently, from Table 4(VI), we have

$$
{ }_{R} H^{(s)} \mathbf{S}_{n}(x, y, 0)={ }_{R} \mathbf{s}_{n}(x, y) .
$$

Now, solving equation (3.5) subject to initial condition (3.7), we get assertion (3.3). Again using a similar argument as in the proof of (3.3), we establish the assertion (3.4).

Theorem 3.3. The following operational representation between the LeGHSP ${ }_{S} H^{(s)} \mathbf{s}_{n}(x, y, z)$ and the $2 V L e S P{ }_{2 L} \mathbf{s}_{n}(x, y)$ holds true:

$$
{ }_{S} H^{(s)} \mathbf{S}_{n}(x, y, z)=\exp \left(z \frac{\partial^{s}}{\partial y^{s}}\right){ }_{2} \mathbf{s}_{n}(x, y) .
$$

Proof. Using a similar argument as in the proof of Theorem 3.2, we establish the assertion (3.8) of Theorem 3.3.

Theorem 3.4. The following operational representation between the LeGHSP ${ }_{R} H^{(s)} \mathbf{s}_{n}(x, y, z)$ and the 2VGLTSP ${ }_{[m]}{ }^{L} \mathbf{s}_{n}(x, y)$ hold true:

$$
{ }_{R} H^{(m)} \mathbf{S}_{n}(x, z, y)=\exp \left(-D_{z}^{-1} \frac{\partial}{\partial D_{x}^{-1}}\right){ }_{[m]}{ }^{L} \mathbf{S}_{n}(x, y) .
$$

Proof. From equations (1.5) and (2.2), we have

$$
\frac{\partial}{\partial D_{z}^{-1}}{ }_{R} H^{(m)} \mathbf{S}_{n}(x, z, y)=-\frac{\partial}{\partial D_{x}^{-1}}{ }_{R} H^{(m)} \mathbf{s}_{n}(x, z, y) .
$$


Since, in view of Table 1 (III), we have

$$
{ }_{R} H_{n}^{(m)}(x, 0, y)={ }_{[m]} L_{n}(x, y) .
$$

Consequently, from Table 4(III), we have

$$
{ }_{R} H^{(m)} \mathbf{S}_{n}(x, 0, y)={ }_{[m]} \mathbf{S}_{n}(x, y) .
$$

Solving equation (3.10) subject to initial condition (3.12), we get assertion (3.9).

Theorem 3.5. The following operational representation between the LeGHSP ${ }_{S} H^{(s)} \mathbf{s}_{n}(x, y, z)$ and the $G H S P_{H^{(s)} \mathbf{S}_{n}}(y, z)$ hold true:

$$
{ }_{S} H^{(s)} \mathbf{S}_{n}(x, y, z)=\exp \left(D_{x}^{-1} \frac{\partial^{2}}{\partial y^{2}}\right){ }_{H^{(s)}} \mathbf{S}_{n}(y, z)
$$

Proof. From equations (1.5) and (2.4), we have

$$
\frac{\partial^{2}}{\partial y^{2}}{ }_{S} H^{(s)} \mathbf{S}_{n}(x, z, y)=\frac{\partial}{\partial D_{x}^{-1}}{ }_{S} H^{(s)} \mathbf{S}_{n}(x, z, y) \text {. }
$$

Since, in view of Table 1(I), we have

$$
{ }_{S} H_{n}^{(s)}(0, y, z)=H_{n}^{(s)}(y, z) .
$$

Consequently, from Table 4(I), we have

$$
{ }_{S} H^{(s)} \mathbf{S}_{n}(0, y, z)={ }_{H^{(s)}} \mathbf{S}_{n}(y, z) .
$$

Solving equation (3.14) subject to initial condition (3.16), we get assertion (3.13).

Next, we prove the integral for the $\operatorname{LeGHSP}_{R} H^{(s)} \mathbf{S}_{n}(x, y, z)$ and ${ }_{S} H^{(s)} \mathbf{s}_{n}(x, y, z)$ in the form of following theorem:

Theorem 3.6. The following integral representations for the LeGHSP ${ }_{R} H^{(s)} \mathbf{s}_{n}(x, y, z)$ and ${ }_{S} H^{(s)} \mathbf{s}_{n}(x, y, z)$ hold true:

$$
{ }_{R} H^{(s)} \mathbf{S}_{n}(x, y, z)=\int_{0}^{\infty} e^{-v}{ }_{R} H^{(s)} \mathbf{S}_{n}\left(x, y, v D_{z}^{-1}\right) d v
$$

and

$$
{ }_{S} H^{(s)} \mathbf{S}_{n}(x, y, z)=\int_{0}^{\infty} e^{-v}{ }_{S} H^{(s)} \mathbf{s}_{n}\left(x, y, v D_{z}^{-1}\right) d v
$$

respectively.

Proof. Using equation (1.1) in the l.h.s. of relation (2.1), we get

$$
\frac{1}{g\left(f^{-1}(t)\right)} \sum_{n=0}^{\infty} \frac{{ }_{R} H_{n}^{(s)}(x, y, z)}{n !} \frac{\left(f^{-1}(t)\right)^{n}}{n !}=\sum_{n=0}^{\infty}{ }_{R} H^{(s)} \mathbf{s}_{n}(x, y, z) \frac{t^{n}}{n !} .
$$


Next, on using the integral representation of LeGHP $\frac{{ }_{R} H_{n}^{(s)}(x, y, z)}{n !}[30]$ :

$$
{ }_{R} H_{n}^{(s)}(x, y, z)=\int_{0}^{\infty} e^{-v}{ }_{R} H_{n}^{(s)}\left(x, y, v D_{z}^{-1}\right) d v
$$

in the l.h.s of equation (3.19) and interchanging the sides, we have

$$
\sum_{n=0}^{\infty}{ }_{R} H^{(s)} \mathbf{s}_{n}(x, y, z) \frac{t^{n}}{n !}=\frac{1}{g\left(f^{-1}(t)\right)} \sum_{n=0}^{\infty}\left(\int_{0}^{\infty} e^{-v}{ }_{R} H_{n}^{(s)}\left(x, y, v D_{z}^{-1}\right) d v\right) \frac{\left(f^{-1}(t)\right)^{n}}{n ! n !},
$$

or, equivalently

$$
\sum_{n=0}^{\infty}{ }_{R} H^{(s)} \mathbf{s}_{n}(x, y, z) \frac{t^{n}}{n !}=\int_{0}^{\infty} e^{-v} \sum_{n=0}^{\infty}\left(\frac{1}{g\left(f^{-1}(t)\right)} \frac{{ }_{R} H_{n}^{(s)}\left(x, y, v D_{z}^{-1}\right)}{n !} \frac{\left(f^{-1}(t)\right)^{n}}{n !}\right) d v .
$$

Again, using equation (3.19) in the r.h.s of the above equation, we find

$$
\sum_{n=0}^{\infty}{ }_{R} H^{(s)} \mathbf{s}_{n}(x, y, z) \frac{t^{n}}{n !}=\int_{0}^{\infty} e^{-v} \sum_{n=0}^{\infty}\left({ }_{R} H^{(s)} \mathbf{s}_{n}\left(x, y, v D_{z}^{-1}\right) \frac{t^{n}}{n !}\right) d v
$$

or, equivalently

$$
\sum_{n=0}^{\infty}{ }_{R} H^{(s)} \mathbf{s}_{n}(x, y, z) \frac{t^{n}}{n !}=\sum_{n=0}^{\infty}\left(\int_{0}^{\infty} e^{-v}{ }_{R} H^{(s)} \mathbf{s}_{n}\left(x, y, v D_{z}^{-1}\right) d v\right) \frac{t^{n}}{n !} .
$$

Finally, equating the coefficient of like powers of $t$ in both sides of the above equation, we get assertion (3.17). Similarly, we can get assertion (3.18)

Corollary 3.1. The following operational representation between the LeGHASP ${ }_{R} H^{(s)} \mathfrak{s}_{n}(x, y, z)$, ${ }_{S} H^{(s)} \mathfrak{s}_{n}(x, y, z)$ and the associated Sheffer polynomials $\mathfrak{s}_{n}(x)$ hold true:

$$
{ }_{R} H^{(s)} \mathfrak{s}_{n}(x, y, z)=\exp \left(z \frac{\partial^{s}}{\partial y^{s}}\right) \mathfrak{s}_{n}\left(-D_{x}^{-1}+D_{y}^{-1}\right)
$$

and

$$
{ }_{S} H^{(s)} \mathfrak{s}_{n}(x, y, z)=\exp \left(D_{x}^{-1} \frac{\partial^{2}}{\partial y^{2}}+z \frac{\partial^{s}}{\partial y^{s}}\right) \mathfrak{s}_{n}(y)
$$

respectively.

Corollary 3.2. The following operational representation between the LeGHASP${ }_{R} H^{(s)} \mathfrak{s}_{n}(x, y, z)$ and the 2 VLeASP ${ }_{R} \mathfrak{s}_{n}(x, y)$ holds true:

$$
{ }_{R} H^{(s)} \mathfrak{s}_{n}(x, y, z)=\exp \left((-1)^{s} z \frac{\partial^{s}}{\partial D_{x}^{-s}}\right) R_{R} \mathfrak{s}_{n}(x, y),
$$

or, equivalently

$$
{ }_{R} H^{(s)} \mathfrak{s}_{n}(x, y, z)=\exp \left(z \frac{\partial^{s}}{\partial D_{y}^{-s}}\right) R_{R}^{\mathfrak{s}_{n}}(x, y) .
$$


Corollary 3.3. The following operational representation between the LeGHASP ${ }_{S H^{(s)}} \mathfrak{s}_{n}(x, y, z)$ and the 2 VLeTASP ${ }_{2} \mathfrak{s}_{n}(x, y)$ holds true:

$$
{ }_{S} H^{(s)} \mathfrak{s}_{n}(x, y, z)=\exp \left(z \frac{\partial^{s}}{\partial y^{s}}\right){ }_{2} \mathfrak{s}_{n}(x, y) .
$$

Corollary 3.4. The following operational representation between the LeGHASP ${ }_{R} H^{(s)} \mathfrak{s}_{n}(x, y, z)$ and the $2 V G L T A S P_{[m]}{ }^{L} \mathfrak{s}_{n}(x, y)$ hold true:

$$
{ }_{R} H^{(m)} \mathfrak{s}_{n}(x, z, y)=\exp \left(-D_{z}^{-1} \frac{\partial}{\partial D_{x}^{-1}}\right){ }_{[m]}{ }^{L} \mathfrak{s}_{n}(x, y) .
$$

Corollary 3.5. The following operational representation between the LeGHASP ${ }_{S} H^{(s)} \mathfrak{s}_{n}(x, y, z)$ and the $G H A S P_{H^{(s)}} \mathfrak{s}_{n}(y, z)$ hold true:

$$
{ }_{S} H^{(s)} \mathfrak{s}_{n}(x, y, z)=\exp \left(D_{x}^{-1} \frac{\partial^{2}}{\partial y^{2}}\right) H^{(s)} \mathfrak{s}_{n}(y, z)
$$

Corollary 3.6. The following integral representations for the $\operatorname{LeGHASP}{ }_{R} H^{(s)} \mathfrak{s}_{n}(x, y, z)$ and ${ }_{S} H^{(s)} \mathfrak{s}_{n}(x, y, z)$ hold true:

$$
{ }_{R} H^{(s)} \mathfrak{s}_{n}(x, y, z)=\int_{0}^{\infty} e^{-v}{ }_{R} H^{(s)} \mathfrak{s}_{n}\left(x, y, v D_{z}^{-1}\right) d v
$$

and

$$
{ }_{S} H^{(s)} \mathfrak{s}_{n}(x, y, z)=\int_{0}^{\infty} e^{-v}{ }_{S} H^{(s)} \mathfrak{s}_{n}\left(x, y, v D_{z}^{-1}\right) d v
$$

respectively.

\section{Examples}

The Sheffer polynomials have been studied because of their remarkable applications not only in different branches of mathematics but also in physics. The Sheffer and associated Sheffer class contains a number of important special polynomials. In this section, some results for the corresponding members of the Legendre-Gould Hopper based Sheffer polynomial families are obtained. We consider the following examples:

Example 4.1: Since, for $A(t)=e^{-t^{r}}$ and $H(t)=v t$, the Sheffer polynomial $\mathbf{s}_{n}(x)$ becomes the generalized Hermite polynomial (GHP) $H_{n, r, v}(x)$ (Table 2(I)). Therefore, for the same choice of $A(t)$ and $H(t)$, the LeGHSP reduce to the Legendre-Gould Hopper based generalized Hermite polynomials (LeGHGHP) ${ }_{R} H^{(s)} H_{n, r, v}(x, y, z)$ and ${ }_{S} H^{(s)} H_{n, r, v}(x, y, z)$.

Thus, In view of equations (2.2), (2.14), (2.18), (2.39), (3.1),(3.3), (3.4), (3.9) and (3.17), we get the following results for the LeGHGHP ${ }_{R} H^{(s)} H_{n, r, v}(x, y, z)$ : 
Table 5. Results for the LeGHGHP ${ }_{R} H^{(s)} H_{n, r, v}(x, y, z)$

\begin{tabular}{|c|c|c|}
\hline I. & Generating functions & $\left.e^{-t^{r}} \exp \left(z(v t)^{s}\right) C_{0}(x v t)\right) C_{0}(-y v t)=\sum_{n=0}^{\infty}{ }^{\infty} H^{(s)} H_{n, r, v}(x, y, z) \frac{t^{n}}{n !}$ \\
\hline II. & $\begin{array}{l}\text { Multiplicative and } \\
\text { derivative operators }\end{array}$ & $\hat{M}:=v\left(-D_{x}^{-1}+D_{y}^{-1}+s z D_{y}^{s-1}\right)-r\left(\frac{1}{v} D_{y} y D_{y}\right)^{r-1}, \quad \hat{P}:=\frac{1}{v} D_{y} y D_{y}$ \\
\hline $\mathrm{III}$ & Differential equations & $\left(\left(\left(-D_{x}^{-1}+D_{y}^{-1}+s z D_{y}^{s-1}\right)\left(D_{y} y D_{y}\right)-r\left(\frac{D_{y} y D_{y}}{v}\right)^{r}\right)-n\right)_{R^{H}(s)} H_{n, r, v}(x, y, z)=0$ \\
\hline $\mathrm{IV}$ & Operational & 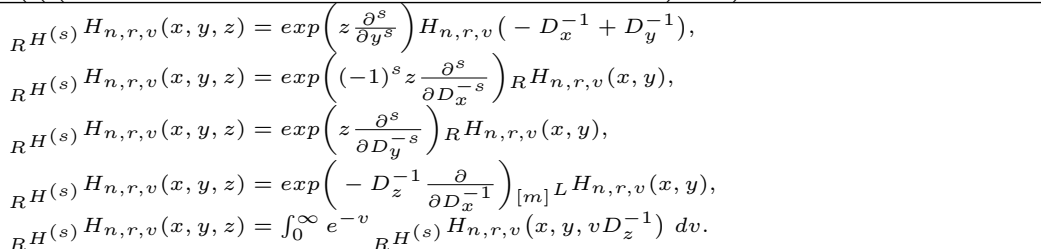 \\
\hline
\end{tabular}

Also, In view of equations (2.4), (2.15), (2.19), (2.41), (3.2),(3.8), (3.13) and (3.18), we get the following results for the LeGHGHP ${ }_{S} H^{(s)} H_{n, r, v}(x, y, z)$ :

Table 6. Results for the LeGHGHP ${ }_{S} H^{(s)} H_{n, r, v}(x, y, z)$

\begin{tabular}{|c|c|c|}
\hline I. & Generating functions & $e^{-t^{r}} \exp \left(y v t+z(v t)^{s}\right) C_{0}\left(-x(v t)^{2}\right)=\sum_{n=0}^{\infty} H_{H}(s) H_{n, r, v}(x, y, z) \frac{t^{n}}{n !}$ \\
\hline II. & $\begin{array}{l}\text { Multiplicative and } \\
\text { derivative operators }\end{array}$ & $\hat{M}:=v\left(y+2 D_{x}^{-1} D_{y}+s z D_{y}^{s-1}\right)-r\left(\frac{1}{v} D_{y}\right)^{r-1}, \quad \hat{P}:=\frac{1}{v} D_{y}$ \\
\hline III & Differential equations & $\left(\left(\left(y+2 D_{x}^{-1} D_{y}+s z D_{y}^{s-1}\right) D_{y}-r\left(\frac{D_{y}}{v}\right)^{r}\right)-n\right)_{S H^{(s)}} H_{n, r, v}(x, y, z)=0$ \\
\hline IV & Operational representations & $\begin{array}{l}{ }_{S} H^{(s)} H_{n, r, v}(x, y, z)=\exp \left(D_{x}^{-1} \frac{\partial^{2}}{\partial y^{2}}+z \frac{\partial^{s}}{\partial y^{s}}\right) H_{n, r, v}(y) \\
S^{H^{(s)}} H_{n, r, v}(x, y, z)=\exp \left(z \frac{\partial^{s}}{\partial y^{s}}\right)_{2} H_{n, r, v}(x, y) \\
S_{S} H^{(s)} H_{n, r, v}(x, y, z)=\exp \left(D_{x}^{-1} \frac{\partial^{2}}{\partial y^{2}}\right)_{H}(s) H_{n, r, v}(y, z) \\
S^{(s)} H_{n, r, v}(x, y, z)=\int_{0}^{\infty} e^{-v}{ }_{S} H^{(s)} H_{n, r, v}\left(x, y, v D_{z}^{-1}\right) d v\end{array}$ \\
\hline
\end{tabular}

Example 4.2: Since, for $A(t)=(1-t)^{-\nu-1}$ and $H(t)=\frac{t}{t-1}$, the Sheffer polynomial $\mathbf{s}_{n}(x)$ becomes the generalized Laguerre polynomial (GHP) $n ! L_{n}^{(\nu)}(x)$ (Table 2(II)). Therefore, for the same choice of $A(t)$ and $H(t)$, the LeGHSP reduce to the Legendre-Gould Hopper based generalized Laguerre polynomials (LeGHGLP) $n !{ }_{R} H^{(s)} L_{n}^{(\nu)}(x, y, z)$ and $n !{ }_{S} H^{(s)} L_{n}^{(\nu)}(x, y, z)$.

Thus, In view of equations (2.2), (2.14), (2.18), (2.39), (3.1),(3.3), (3.4), (3.9) and (3.17), we get the following results for the LeGHGLP $n !{ }_{R} H^{(s)} L_{n}^{(\nu)}(x, y, z)$ :

Table 7. Results for the LeGHGLP $n !{ }_{R} H^{(s)} L_{n}^{(\nu)}(x, y, z)$

\begin{tabular}{|c|c|c|}
\hline I. & Generating functions & $(1-t)^{-\nu-1} \exp \left(z\left(\frac{t}{t-1}\right)^{s}\right) C_{0}\left(\frac{x t}{t-1}\right) C_{0}\left(-\frac{y t}{t-1}\right)=\sum_{n=0}^{\infty} R^{(s)} L_{n}^{(\nu)}(x, y, z) \frac{t^{n}}{n !}$ \\
\hline II. & $\begin{array}{l}\text { Multiplicative and } \\
\text { derivative operators }\end{array}$ & $\hat{M}:=-\left(-D_{x}^{-1}+D_{y}^{-1}+s z D_{y}^{s-1}\right)\left(D_{y} y D_{y}-1\right)^{2}+(\nu+1)\left(1-D_{y} y D_{y}\right), \quad \hat{P}:=\frac{D_{y} y D_{y}}{D_{y} y D_{y}-1}$ \\
\hline III & Differential equations & $\left(\left(\left(-D_{x}^{-1}+D_{y}^{-1}+s z D_{y}^{s-1}\right)\left(\left(D_{y} y D_{y}\right)^{2}-D_{y} y D_{y}\right)-(\nu+1)\left(D_{y} y D_{y}\right)\right)-n\right)_{R^{H}(s)} L_{n}^{(\nu)}(x, y, z)=0$ \\
\hline IV & $\begin{array}{l}\text { Operational } \\
\text { representations }\end{array}$ & $\begin{array}{l}R_{R}^{H^{(s)}} L_{n}^{(\nu)}(x, y, z)=\exp \left(z \frac{\partial^{s}}{\partial y^{s}}\right) L_{n}^{(\nu)}\left(-D_{x}^{-1}+D_{y}^{-1}\right),{ }_{R} H^{(s)} L_{n}^{(\nu)}(x, y, z)=\exp \left((-1)^{s} z \frac{\partial^{s}}{\partial D_{x}^{-s}}\right){ }_{R} L_{n}^{(\nu)}(x, y), \\
R^{H^{(s)}} L_{n}^{\nu}(x, y, z)=\exp \left(z \frac{\partial^{s}}{\partial D_{y}^{-s}}\right) R_{R} L_{n}^{(\nu)}(x, y),{ }_{R} H^{(s)} L_{n}^{\nu}(x, y, z)=\exp \left(-D_{z}^{-1} \frac{\partial}{\partial D_{x}^{-1}}\right)_{[m]} L_{n} L_{n}^{(\nu)}(x, y), \\
{ }_{R} H^{(s)} L_{n}^{(\nu)}(x, y, z)=\int_{0}^{\infty} e^{-v}{ }_{R^{H}}(s) L_{n}^{(\nu)}\left(x, y, v D_{z}^{-1}\right) d v .\end{array}$ \\
\hline
\end{tabular}

Also, In view of equations (2.4), (2.15), (2.19), (2.41), (3.2),(3.8), (3.13) and (3.18), we get the following results for the LeGHGLP ${ }_{S} H^{(s)} L_{n}^{(\nu)}(x, y, z)$ : 
Table 8. Results for the LeGHGLP ${ }_{S} H^{(s)} L_{n}^{(\nu)}(x, y, z)$

\begin{tabular}{|c|c|c|}
\hline I. & Generating functions & $(1-t)^{-\nu-1} \exp \left(\frac{y t}{t-1}+z\left(\frac{t}{t-1}\right)^{s}\right) C_{0}\left(-x\left(\frac{t}{t-1}\right)^{2}\right)=\sum_{n=0 S}^{\infty} H^{(s)} L_{n}^{(\nu)}(x, y, z) \frac{t^{n}}{n !}$ \\
\hline II. & $\begin{array}{l}\text { Multiplicative and } \\
\text { derivative operators }\end{array}$ & $\hat{M}:=-\left(y+2 D_{x}^{-1} D_{y}+s z D_{y}^{s-1}\right)\left(D_{y}-1\right)^{2}+(\nu+1)\left(1-D_{y}\right), \quad \hat{P}:=\frac{D_{y}}{D_{y}-1}$ \\
\hline III & Differential equations & $\left(\left(\left(y+2 D_{x}^{-1} D_{y}+s z D_{y}^{s-1}\right)\left(D_{y}^{2}-D_{y}\right)-(\nu+1) D_{y}\right)-n\right)_{S H^{(s)}} L_{n}^{(\nu)}(x, y, z)=0$ \\
\hline IV & Operational representations & $\begin{array}{l}S_{S}^{(s)} L_{n}^{(\nu)}(x, y, z)=\exp \left(D_{x}^{-1} \frac{\partial^{2}}{\partial y^{2}}+z \frac{\partial^{s}}{\partial y^{s}}\right) L_{n}^{(\nu)}(y), \\
S^{H^{(s)}} L_{n}^{(\nu)}(x, y, z)=\exp \left(z \frac{\partial^{s}}{\partial y^{s}}\right)_{2} L_{n}^{(\nu)}(x, y) \\
S_{S} H^{(s)} L_{n}^{(\nu)}(x, y, z)=\exp \left(D_{x}^{-1} \frac{\partial^{2}}{\partial y^{2}}\right)_{H^{(s)}} L_{n}^{(\nu)}(y, z), \\
{ }_{S} H^{(s)} L_{n}^{(\nu)}(x, y, z)=\int_{0}^{\infty} e^{-v}{ }_{S} H^{(s)} L_{n}^{(\nu)}\left(x, y, v D_{z}^{-1}\right) d v .\end{array}$ \\
\hline
\end{tabular}

Example 4.3: Since, for $A(t)=\frac{t}{1-t}$ and $H(t)=\ln \left(\frac{1+t}{1-t}\right)$, the Sheffer polynomial $\mathbf{s}_{n}(x)$ becomes the Pidduck polynomial (PP) $P_{n}(x)$ (Table 2(III)). Therefore, for the same choice of $A(t)$ and $H(t)$, the LeGHSP reduce to the Legendre-Gould Hopper based Pidduck polynomials (LeGHPP) ${ }_{R} H^{(s)} P_{n}(x, y, z)$ and ${ }_{S} H^{(s)} P_{n}(x, y, z)$.

Thus, In view of equations (2.2), (2.14), (2.18), (2.39), (3.1),(3.3), (3.4), (3.9) and (3.17), we get the following results for the LeGHPP ${ }_{R} H^{(s)} P_{n}(x, y, z)$ :

Table 9. Results for the LeGHPP ${ }_{R} H^{(s)} P_{n}(x, y, z)$

\begin{tabular}{|c|c|c|}
\hline I. & Generating functions & $\frac{t}{1-t} \exp \left(z\left(\ln \left(\frac{1+t}{1-t}\right)\right)^{s}\right) C_{0}\left(x \ln \left(\frac{1+t}{1-t}\right)\right) C_{0}\left(-y \ln \left(\frac{1+t}{1-t}\right)\right)=\sum_{n=0}^{\infty}{ }^{\infty} H^{(s)} P_{n}(x, y, z) \frac{t^{n}}{n !}$ \\
\hline II. & $\begin{array}{l}\text { Multiplicative and } \\
\text { derivative operators }\end{array}$ & $\hat{M}:=\left(-D_{x}^{-1}+D_{y}^{-1}+s z D_{y}^{s-1}\right)\left(\frac{\left(e^{D_{y} y D_{y}}+1\right)^{2}}{2 e^{D y y D}}\right)+\frac{\left(e^{D_{y} y D_{y}}+1\right)^{2}}{2\left(e^{D y y D y}-1\right)}, \quad \hat{P}:=\frac{e^{D y y D} e_{-1}}{e^{D y y D}+1}$ \\
\hline III & Differential equations & $\left(\left(\left(-D_{x}^{-1}+D_{y}^{-1}+s z D_{y}^{s-1}\right)\left(\frac{e^{2 D_{y} y D_{y}-1}}{2 e^{D y} D_{y}}\right)+\frac{1}{2}\left(e^{D_{y} y D_{y}}+1\right)\right)-n\right)_{R}(s) P_{n}(x, y, z)=0$ \\
\hline $\mathrm{IV}$ & $\begin{array}{l}\text { Operational } \\
\text { representations }\end{array}$ & $\begin{array}{l}R^{H(s)} P_{n}(x, y, z)=\exp \left(z \frac{\partial^{s}}{\partial y^{s}}\right) P_{n}\left(-D_{x}^{-1}+D_{y}^{-1}\right),{ }_{R} H^{(s)} P_{n}(x, y, z)=\exp \left((-1)^{s} z \frac{\partial^{s}}{\partial D_{x}^{-s}}\right){ }_{R} P_{n}(x, y), \\
{ }_{R^{H}}(s) P_{n}(x, y, z)=\exp \left(z \frac{\partial^{s}}{\partial D_{y}^{-s}}\right){ }_{R} P_{n}(x, y),{ }_{R}{ }^{(s)} P_{n}(x, y, z)=\exp \left(-D_{z}^{-1} \frac{\partial}{\partial D_{x}^{-1}}\right)_{[m]} P_{n}(x, y), \\
R^{H^{(s)}} P_{n}(x, y, z)=\int_{0}^{\infty} e^{-v}{ }_{R^{H}}{ }^{(s)} P_{n}\left(x, y, v D_{z}^{-1}\right) d v .\end{array}$ \\
\hline
\end{tabular}

Also, In view of equations (2.4), (2.15), (2.19), (2.41), (3.2),(3.8), (3.13) and (3.18), we get the following results for the $\operatorname{LeGHPP}_{S} H^{(s)} P_{n}(x, y, z)$ :

Table 10. Results for the LeGHPP ${ }_{S} H^{(s)} P_{n}(x, y, z)$

\begin{tabular}{|c|c|c|}
\hline I. & Generating functions & $\frac{t}{1-t} \exp \left(y \ln \left(\frac{1+t}{1-t}\right)+z\left(\ln \left(\frac{1+t}{1-t}\right)\right)^{s}\right) C_{0}\left(-x\left(\ln \left(\frac{1+t}{1-t}\right)\right)^{2}\right)=\sum_{n=0}^{\infty}{ }_{S} H^{(s)} P_{n}(x, y, z) \frac{t^{n}}{n !}$ \\
\hline II. & $\begin{array}{l}\text { Multiplicative and } \\
\text { derivative operators }\end{array}$ & $\hat{M}:=-\left(y+2 D_{x}^{-1} D_{y}+s z D_{y}^{s-1}\right)\left(\frac{\left(e^{D y}+1\right)^{2}}{2 e^{D y}}\right)+\frac{\left(e^{D y}+1\right)^{2}}{2\left(e^{D y}-1\right)}, \quad \hat{P}:=\frac{e^{D y}-1}{e^{D y}+1}$ \\
\hline III & Differential equations & $\left(\left(\left(y+2 D_{x}^{-1} D_{y}+s z D_{y}^{s-1}\right)\left(\frac{e^{2 D y}-1}{2 e^{D y}}\right)+\frac{1}{2}\left(e^{D y}+1\right)\right)-n\right)_{S H}(s) P_{n}(x, y, z)=0$ \\
\hline IV & Operational representations & 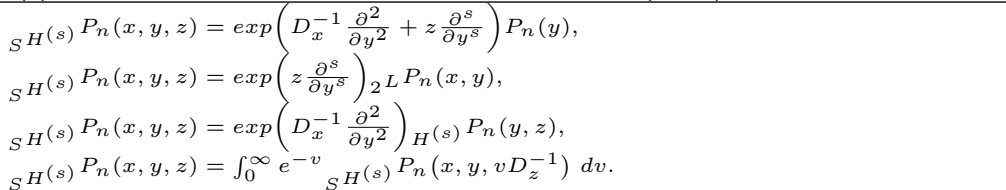 \\
\hline
\end{tabular}

Example 4.4: Since, for $H(t)=\ln \left(\frac{1+t}{1-t}\right)$, the associated Sheffer polynomial $\mathfrak{s}_{n}(x)$ becomes the Mittag-Leffler polynomial (MLP) $M_{n}(x)$ (Table 3(I)). Therefore, for the same choice of $H(t)$, the LeGHASP reduce to the Legendre-Gould Hopper based Mittag-Leffler polynomials (LeGHMLP) ${ }_{R} H^{(s)} M_{n}(x, y, z)$ and ${ }_{S} H^{(s)} M_{n}(x, y, z)$. 
Thus, in view of equations (2.43), (2.48), (2.52), (2.55), (3.25),(3.27), (3.28), (3.30) and (3.32), we get the following results for the LeGHMLP ${ }_{R} H^{(s)} M_{n}(x, y, z)$ :

Table 11. Results for the LeGHMLP ${ }_{R} H^{(s)} M_{n}(x, y, z)$

\begin{tabular}{|c|c|c|}
\hline I. & Generating functions & $\exp \left(z\left(\ln \left(\frac{1+t}{1-t}\right)\right)^{s}\right) C_{0}\left(x \ln \left(\frac{1+t}{1-t}\right)\right) C_{0}\left(-y \ln \left(\frac{1+t}{1-t}\right)\right)=\sum_{n=0}^{\infty} R^{\infty}(s) M_{n}(x, y, z) \frac{t^{n}}{n !}$ \\
\hline II. & $\begin{array}{l}\text { Multiplicative and } \\
\text { derivative operators }\end{array}$ & $\hat{M}:=\left(-D_{x}^{-1}+D_{y}^{-1}+s z D_{y}^{s-1}\right)\left(\frac{\left(e^{D_{y} y D_{y}+1}\right)^{2}}{2 e^{D y y D_{y}}}\right), \quad \hat{P}:=\frac{e^{D_{y} y D_{y}-1}}{e^{D_{y} y D_{y}+1}}$ \\
\hline III & Differential equations & $\left(\left(-D_{x}^{-1}+D_{y}^{-1}+s z D_{y}^{s-1}\right)\left(\frac{e^{2 D_{y} y D_{y}-1}}{2 e^{D} D_{y} D_{y}}\right)-n\right)_{R} H^{(s)} M_{n}(x, y, z)=0$ \\
\hline IV & $\begin{array}{l}\text { Operational } \\
\text { representations }\end{array}$ & $\begin{array}{l}R_{R^{(s)}} M_{n}(x, y, z)=\exp \left(z \frac{\partial^{s}}{\partial y^{s}}\right) M_{n}\left(-D_{x}^{-1}+D_{y}^{-1}\right),{ }_{R} H^{(s)} M_{n}(x, y, z)=\exp \left((-1)^{s} z \frac{\partial^{s}}{\partial D_{x}^{-s}}\right){ }_{R} M_{n}(x, y), \\
R^{H^{(s)}} M_{n}(x, y, z)=\exp \left(z \frac{\partial^{s}}{\partial D_{y}^{-s}}\right){ }_{R} M_{n}(x, y),{ }_{R} H^{(s)} M_{n}(x, y, z)=\exp \left(-D_{z}^{-1} \frac{\partial}{\partial D_{x}^{-1}}\right){ }_{[m]}{ }^{L} M_{n}(x, y), \\
{ }_{R} H^{(s)} M_{n}(x, y, z)=\int_{0}^{\infty} e^{-v}{ }_{R^{(s)}} M_{n}\left(x, y, v D_{z}^{-1}\right) d v .\end{array}$ \\
\hline
\end{tabular}

Also, in view of equations (2.45), (2.49), (2.53), (2.57), (3.26),(3.29), (3.31) and (3.33), we get the following results for the LeGHMLP ${ }_{S} H^{(s)} M_{n}(x, y, z)$ :

Table 12. Results for the LeGHMLP ${ }_{S} H^{(s)} M_{n}(x, y, z)$

\begin{tabular}{|c|c|c|}
\hline I. & Generating functions & $\exp \left(y \ln \left(\frac{1+t}{1-t}\right)+z\left(\ln \left(\frac{1+t}{1-t}\right)\right)^{s}\right) C_{0}\left(-x\left(\ln \left(\frac{1+t}{1-t}\right)\right)^{2}\right)=\sum_{n=0}^{\infty} H^{(s)} M_{n}(x, y, z) \frac{t^{n}}{n !}$ \\
\hline II. & $\begin{array}{l}\text { Multiplicative and } \\
\text { derivative operators }\end{array}$ & $\hat{M}:=-\left(y+2 D_{x}^{-1} D_{y}+s z D_{y}^{s-1}\right)\left(\frac{\left(e^{D y}+1\right)^{2}}{2 e^{D y}}\right), \quad \hat{P}:=\frac{e^{D y}-1}{e^{D y}+1}$ \\
\hline III & Differential equations & $\left(\left(y+2 D_{x}^{-1} D_{y}+s z D_{y}^{s-1}\right)\left(\frac{e^{2 D y}-1}{2 e^{D} y}\right)-n\right)_{S} H^{(s)} M_{n}(x, y, z)=0$ \\
\hline IV & Operational representations & $\begin{array}{l}{ }_{S}{ }^{(s)} M_{n}(x, y, z)=\exp \left(D_{x}^{-1} \frac{\partial^{2}}{\partial y^{2}}+z \frac{\partial^{s}}{\partial y^{s}}\right) M_{n}(y) \\
{ }_{S}{ }^{(s)} M_{n}(x, y, z)=\exp \left(z \frac{\partial^{s}}{\partial y^{s}}\right)_{2} M_{n}(x, y) \\
{ }_{S} H^{(s)} M_{n}(x, y, z)=\exp \left(D_{x}^{-1} \frac{\partial^{2}}{\partial y^{2}}\right)_{H(s)} M_{n}(y, z) \\
{ }_{S} H^{(s)} M_{n}(x, y, z)=\int_{0}^{\infty} e^{-v}{ }_{S} H^{(s)} M_{n}\left(x, y, v D_{z}^{-1}\right) d v\end{array}$ \\
\hline
\end{tabular}

Example 4.5: Since, for $H(t)=e^{t}-1$, the associated Sheffer polynomial $\mathfrak{s}_{n}(x)$ becomes the Exponential polynomial (EP) $\varphi_{n}(x)$ (Table 3(II)). Therefore, for the same choice of $H(t)$, the LeGHASP reduce to the Legendre-Gould Hopper based Exponential polynomials (LeGHEP) ${ }_{R} H^{(s)} \varphi_{n}(x, y, z)$ and ${ }_{S} H^{(s)} \varphi_{n}(x, y, z)$.

Thus, in view of equations (2.43), (2.48), (2.52), (2.55), (3.25),(3.27), (3.28), (3.30) and (3.32), we get the following results for the $\operatorname{LeGHEP}_{R} H^{(s)} \varphi_{n}(x, y, z)$ :

Table 13. Results for the LeGHEP ${ }_{R} H^{(s)} \varphi_{n}(x, y, z)$

\begin{tabular}{|r|l|l|}
\hline I. & Generating functions & $\exp \left(z\left(e^{t}-1\right)^{s}\right) C_{0}\left(x\left(e^{t}-1\right)\right) C_{0}\left(-y\left(e^{t}-1\right)\right)=\sum_{n=0}^{\infty} H^{(s)} \varphi_{n}(x, y, z) \frac{t^{n}}{n !}$ \\
\hline II. & $\begin{array}{l}\text { Multiplicative and } \\
\text { derivative operators }\end{array}$ & $\hat{M}:=\left(-D_{x}^{-1}+D_{y}^{-1}+s z D_{y}^{s-1}\right)\left(1+D_{y} y D_{y}\right), \hat{P}:=\ln \left(1+D_{y} y D_{y}\right)$ \\
\hline III & Differential equations & $\left(\left(-D_{x}^{-1}+D_{y}^{-1}+s z D_{y}^{s-1}\right)\left(1+D_{y} y D_{y}\right) \ln \left(1+D_{y} y D_{y}\right)-n\right)_{R^{H}}(s) \varphi_{n}(x, y, z)=0$ \\
\hline IV & Operational & $\begin{array}{l}R^{H^{(s)}} \varphi_{n}(x, y, z)=\exp \left(z \frac{\partial^{s}}{\partial y^{s}}\right) \varphi_{n}\left(-D_{x}^{-1}+D_{y}^{-1}\right),{ }_{R} H^{(s)} \varphi_{n}(x, y, z)=\exp \left((-1)^{s} z \frac{\partial^{s}}{\partial D_{x}^{-s}}\right) r_{R} \varphi_{n}(x, y), \\
R^{H^{(s)}} \varphi_{n}(x, y, z)=\exp \left(z \frac{\partial^{s}}{\partial D_{y}^{-s}}\right) R \varphi_{n}(x, y){ }_{R^{H}(s)} \varphi_{n}(x, y, z)=\exp \left(-D_{z}^{-1} \frac{\partial}{\partial D_{x}^{-1}}\right)_{[m]} \varphi_{n}(x, y), \\
R^{H^{(s)}} \varphi_{n}(x, y, z)=\int_{0}^{\infty} e_{R^{H}}^{-v} \varphi_{n}\left(x, y, v D_{z}^{-1}\right) d v .\end{array}$ \\
\hline
\end{tabular}

Also, in view of equations (2.45), (2.49), (2.53), (2.57), (3.26),(3.29), (3.31) and (3.33), we get the following results for the $\operatorname{LeGHEP}_{S} H^{(s)} \varphi_{n}(x, y, z)$ : 
Table 14. Results for the LeGHEP ${ }_{S} H^{(s)} \varphi_{n}(x, y, z)$

\begin{tabular}{|c|c|c|}
\hline I. & Generating functions & $\exp \left(y\left(e^{t}-1\right)+z\left(e^{t}-1\right)^{s}\right) C_{0}\left(-x\left(e^{t}-1\right)^{2}\right)=\sum_{n=0}^{\infty}{ }_{n} H^{(s)} \varphi_{n}(x, y, z) \frac{t^{n}}{n !}$ \\
\hline II. & $\begin{array}{l}\text { Multiplicative and } \\
\text { derivative operators }\end{array}$ & $\hat{M}:=-\left(y+2 D_{x}^{-1} D_{y}+s z D_{y}^{s-1}\right)\left(1+D_{y}\right), \quad \hat{P}:=\ln \left(1+D_{y}\right)$ \\
\hline III & Differential equations & $\left(\left(y+2 D_{x}^{-1} D_{y}+s z D_{y}^{s-1}\right)\left(1+D_{y}\right) \ln \left(1+D_{y}\right)-n\right)_{S H}(s) \varphi_{n}(x, y, z)=0$ \\
\hline IV & $\begin{array}{l}\text { Operational } \\
\text { representations }\end{array}$ & $\begin{array}{l}S_{S}{ }^{(s)} \varphi_{n}(x, y, z)=\exp \left(D_{x}^{-1} \frac{\partial^{2}}{\partial y^{2}}+z \frac{\partial^{s}}{\partial y^{s}}\right) \varphi_{n}(y),{ }_{S} H^{(s)} \varphi_{n}(x, y, z)=\exp \left(z \frac{\partial^{s}}{\partial y^{s}}\right)_{2} \varphi_{n}(x, y), \\
{ }_{S} H^{(s)} \varphi_{n}(x, y, z)=\exp \left(D_{x}^{-1} \frac{\partial^{2}}{\partial y^{2}}\right)_{H^{(s)} \varphi_{n}(y, z),}{ }_{S} H^{(s)} \varphi_{n}(x, y, z)=\int_{0}^{\infty} e^{-v}{ }_{S} H^{(s)} \varphi_{n}\left(x, y, v D_{z}^{-1}\right) d v .\end{array}$ \\
\hline
\end{tabular}

Similarly, for the other members of the Sheffer and associated families (see Tables 2 and 3), there exist new special polynomials belonging to the LeGHSP and LeGHASP families respectively. The generating functions and other properties of these special polynomials can be obtained from the results derived in the second and third sections.

\section{Concluding Remarks}

A determinantal definition for the classical Bernoulli polynomials introduced by Costabile et.al. [10] has given a new approach to Bernoulli polynomials which was further extended to provide the determinantal definition of the Appell polynomials [11]. Recently, the determinantal definition of Appell sequences is extended to Sheffer sequences by using the theory of Riordan arrays [29].

The determinantal approach considered in $[10,11,29]$ provides motivation to consider the determinantal form of the new families of special polynomials. In this section, we give the determinantal definitions for the Legendre-Gould Hopper based Sheffer polynomials ${ }_{R} H^{(s)} \mathbf{S}_{n}(x, y, z)$ and ${ }_{S} H^{(s)} \mathbf{S}_{n}(x, y, z)$ as:

Definition 5.1. The Legendre-Gould Hopper based Sheffer polynomials ${ }_{S} H^{(s)} \mathbf{s}_{n}(x, y, z)$ of degree $n$ are defined by

$$
\begin{aligned}
{ }_{S} H^{(s)} \mathbf{s}_{0}(x, y, z) & =\frac{1}{\alpha_{0,0}}, \\
{ }_{S} H^{(s)} \mathbf{s}_{n}(x, y, z) & =\frac{(-1)^{n}}{\alpha_{0,0} \alpha_{1,1} \ldots \alpha_{n, n}} \\
& \left|\begin{array}{cccccc}
1 & { }_{S} H_{1}^{(s)}(x, y, z) & { }_{S} H_{2}^{(s)}(x, y, z) & \ldots & { }_{S} H_{n-1}^{(s)}(x, y, z) & { }_{S} H_{n}^{(s)}(x, y, z) \\
\alpha_{0,0} & \alpha_{1,0} & \alpha_{2,0} & \ldots & \alpha_{n-1,0} & \alpha_{n, 0} \\
0 & \alpha_{1,1} & \alpha_{2,1} & \ldots & \alpha_{n-1,1} & \alpha_{n, 1} \\
0 & 0 & \alpha_{2,2} & \ldots & \alpha_{n-1,2} & \alpha_{n, 1} \\
\cdot & \cdot & \cdots & \cdot & \cdot \\
\cdot & \cdot & \cdots & \cdot & \cdot \\
0 & 0 & & \ldots & \alpha_{n-1, n-1} & \alpha_{n, n-1}
\end{array}\right|, \\
& =\frac{(-1)^{n}}{\alpha_{0,0} \alpha_{1,1} \ldots \alpha_{n, n}} \operatorname{det}\left(\begin{array}{c}
\mathrm{H}_{n+1}(x, y, z) \\
\mathrm{S}_{n \times(n+1)}
\end{array}\right),
\end{aligned}
$$


where $\mathrm{H}_{n+1}(x, y, z)=\left(1,{ }_{S} H_{1}^{(s)}(x, y, z),{ }_{S} H_{2}^{(s)}(x, y, z), \ldots,{ }_{S} H_{n}^{(s)}(x, y, z)\right)$ and ${ }_{S} H_{n}^{(s)}(x, y, z)(n=$ $0,1,2, \ldots$,$) are the Legendre-Gould Hopper polynomials defined by equation (1.2), \mathrm{S}_{n \times(n+1)}=$ $\left(a_{j-1, i-1}\right) 1 \leq i \leq n, 1 \leq j \leq n+1$ and $\alpha_{n, k}$ is the $(n, k)$ entry of the Riordan array $(g(t), f(t))$.

Definition 5.1. The Legendre-Gould Hopper based Sheffer polynomials ${ }_{R} H^{(s)} \mathbf{s}_{n}(x, y, z)$ of degree $n$ are defined by

$$
\begin{aligned}
& { }_{R} H^{(s)} \mathbf{S}_{0}(x, y, z)=\frac{1}{\alpha_{0,0}}, \\
& { }_{R} H^{(s)} \mathbf{s}_{n}(x, y, z)=\frac{(-1)^{n}}{\alpha_{0,0} \alpha_{1,1} \ldots \alpha_{n, n}} \\
& \left|\begin{array}{cccccc}
1 & \frac{{ }_{R} H_{1}^{(s)}(x, y, z)}{1 !} & \frac{{ }_{R} H_{2}^{(s)}(x, y, z)}{2 !} & \ldots & \frac{{ }_{R} H_{n-1}^{(s)}(x, y, z)}{(n-1) !} & \frac{{ }_{R} H_{n}^{(s)}(x, y, z)}{n !} \\
\alpha_{0,0} & \alpha_{1,0} & \alpha_{2,0} & \ldots & \alpha_{n-1,0} & \alpha_{n, 0} \\
0 & \alpha_{1,1} & \alpha_{2,1} & \ldots & \alpha_{n-1,1} & \alpha_{n, 1} \\
0 & 0 & \alpha_{2,2} & \ldots & \alpha_{n-1,2} & \alpha_{n, 1} \\
\cdot & \cdot & \cdot & \ldots & \cdot & \cdot \\
\cdot & \cdot & \cdot & \ldots & \cdot & \cdot \\
0 & 0 & 0 & \ldots & \alpha_{n-1, n-1} & \alpha_{n, n-1}
\end{array}\right|, \\
& =\frac{(-1)^{n}}{\alpha_{0,0} \alpha_{1,1} \ldots \alpha_{n, n}} \operatorname{det}\left(\begin{array}{c}
H_{n+1}(x, y, z) \\
\mathbf{S}_{n \times(n+1)}
\end{array}\right) \text {, }
\end{aligned}
$$

where $H_{n+1}(x, y, z)=\left(1, \frac{{ }_{R} H_{1}^{(s)}(x, y, z)}{1 !}, \frac{{ }_{R} H_{2}^{(s)}(x, y, z)}{2 !}, \ldots, \frac{{ }_{R} H_{n}^{(s)}(x, y, z)}{n !}\right)$ and $\frac{{ }_{R} H_{n}^{(s)}(x, y, z)}{n !}(n=0,1,2, \ldots$, are the Legendre-Gould Hopper polynomials defined by equation (1.1), $\mathrm{S}_{n \times(n+1)}=\left(\alpha_{j-1, i-1}\right) 1 \leq$ $i \leq n, 1 \leq j \leq n+1$ and $a_{n, k}$ is the $(n, k)$ entry of the Riordan array $(g(t), f(t))$.

Further, we consider an example of mixed families of special polynomials belonging to Legendre-Gould Hopper based Sheffer polynomials.

In [29], it has been shown that for $\alpha_{n, k}=(-1)^{k} \frac{n !}{k !}\left(\begin{array}{l}n+\nu \\ n-k\end{array}\right)$ the determinantal definition of the Sheffer polynomials reduces to the determinantal definition of the Laguerre polynomials of order $\nu, L_{n}^{(\nu)}(x)$. Therefore, on taking $\mathbf{s}_{n}(x)=L_{n}^{(\nu)}(x)$ and $\alpha_{n, k}=(-1)^{k} \frac{n !}{k !}\left(\begin{array}{c}n+\nu \\ n-k\end{array}\right)$ in equations (5.3) and (5.4), the determinantal definition of Legendre-Gould Hopper based generalized Laguerre polynomials $n !{ }_{R} H^{(s)} L_{n}^{(\nu)}(x, y, z)$ is given as:

Definition 5.1. The Legendre-Gould Hopper based generalized Laguerre polynomials ${ }_{R} H^{(s)} L_{n}^{(\nu)}(x, y, z)$ of degree $n$ are defined by

$$
{ }_{R} H^{(s)} L_{0}^{(\nu)}(x, y, z)=1,
$$




$$
\begin{aligned}
& { }_{R} H^{(s)} L_{n}^{(\nu)}(x, y, z)=\frac{(-1)^{n}}{\alpha_{0,0} \alpha_{1,1} \ldots \alpha_{n, n}} \\
& \left|\begin{array}{cccccc}
1 & \frac{{ }_{R} H_{1}^{(s)}(x, y, z)}{1 !} & \frac{{ }_{R} H_{2}^{(s)}(x, y, z)}{2 !} & \ldots & \frac{{ }_{R} H_{n-1}^{(s)}(x, y, z)}{(n-1) !} & \frac{{ }_{R} H_{n}^{(s)}(x, y, z)}{n !} \\
1 & \nu+1 & (\nu+2)_{2} & \ldots & (\nu+n-1)_{n-1} & (\nu+n)_{n} \\
0 & -1 & -2(\nu+2) & \ldots & -(n-1)(\nu+n-1)_{n-2} & -n(\nu+n)_{n-1} \\
0 & 0 & 1 & \ldots & \frac{(n-1)(n-2)}{2}(\nu+n-1)_{n-3} & \frac{n(n-1)}{2}(\nu+n)_{n-2} \\
. & . & . & \ldots & . & \cdot \\
0 & 0 & 0 & \ldots & (-1)^{n-1} & (-1)^{n-1} n(\nu+n)
\end{array}\right|, \\
& =(-1)^{\frac{n(n+3)}{2}} \operatorname{det}\left(\begin{array}{c}
H_{n+1}(x, y, z) \\
\mathrm{S}_{n \times(n+1)}
\end{array}\right) \text {, }
\end{aligned}
$$

where $H_{n+1}(x, y, z)=\left(1, \frac{{ }_{R} H_{1}^{(s)}(x, y, z)}{1 !}, \frac{{ }_{R} H_{2}^{(s)}(x, y, z)}{2 !}, \ldots, \frac{{ }_{R} H_{n}^{(s)}(x, y, z)}{n !}\right)$ and $\frac{{ }_{R} H_{n}^{(s)}(x, y, z)}{n !}(n=0,1,2, \ldots$, are the Legendre-Gould Hopper polynomials defined by equation (1.1), $\mathrm{S}_{n \times(n+1)}=\left(\alpha_{j-1, i-1}\right) 1 \leq$ $i \leq n, 1 \leq j \leq n+1$ and $a_{n, k}$ is the $(n, k)$ entry of the Riordan array $(g(t), f(t))$.

Also, by giving suitable values to the variables and indices we can find the determinantal definitions of the other members belonging to the Legendre-Gould Hopper based Sheffer family. We conclude this paper with a further remark concerning the possibility of extending the method to other generalized special polynomials.

\section{References}

[1] L. C. Andrews. Special functions for engineers and applied mathematicians. Macmillan, 1985 .

[2] P. Appell and J. Kampé de Fériet. Fonctions Hypergéométriques et Hypersphériques; Polynômes d'Hermite. Gauthier-Villars, Paris, 1926.

[3] H. Bateman. The polynomial of mittag-leffler. Proceedings of the National Academy of Sciences, 26(8):491-496, 1940.

[4] H. Bateman. Manuscript project. In Higher transcendental functions, volume III. McGraw-Hill Toulouse, 1955.

[5] H. Bateman, A. Erdélyi, W. Magnus, F. Oberhettinger, and F. G. Tricomi. Higher transcendental functions, volume II. McGraw-Hill New York, 1955.

[6] E. T. Bell. Exponential polynomials. Annals of Mathematics, pages 258-277, 1934. 
[7] C. M. Bender. Solution of operator equations of motion. J. Dittrich, P. Exner (Eds.), Rigorous Results in Quantum Dynamics, World Scientific, Singapore, pages 99-112, 1991.

[8] R. P. Boas and R. C. Buck. Polynomial expansions of analytic functions. SpringerVerlag, Berlin, Gottingen, Heidelberg, 1958.

[9] L. Carlitz et al. A note on the bessel polynomials. Duke Mathematical Journal, 24(2):151-162, 1957.

[10] F. Costabile, F. Dell'Accio, and M. Gualtieri. A new approach to Bernoulli polynomials. Rend. Mat. Appl, 26(1):1-12, 2006.

[11] F. A. Costabile and E. Longo. A determinantal approach to appell polynomials. Journal of computational and applied mathematics, 234(5):1528-1542, 2010.

[12] G. Dattoli. Hermite-Bessel and Laguerre-Bessel functions: A by-product of the monomiality principle. Advanced special functions and applications (Melfi, 1999), 147-164. In Proc. Melfi Sch. Adv. Top. Math. Phys, volume 1.

[13] G. Dattoli. Integral transforms and chebyshev-like polynomials. Applied mathematics and computation, 148(1):225-234, 2004.

[14] G. Dattoli, C. Cesarano, and D. Sacchetti. A note on truncated polynomials. Applied Mathematics and Computation, 134(2):595-605, 2003.

[15] G. Dattoli, B. Germano, M. Martinelli, and P. Ricci. Monomiality, orthogonal and pseudo-orthogonal polynomials. In Int. Math. Forum, volume 1, pages 603-616. Citeseer, 2006.

[16] G. Dattoli, B. Germano, and P. E. Ricci. Comments on monomiality, ordinary polynomials and associated bi-orthogonal functions. Applied mathematics and computation, 154(1):219-227, 2004.

[17] G. Dattoli, S. Lorenzutta, A. Mancho, and A. Torre. Generalized polynomials and associated operational identities. Journal of computational and applied mathematics, 108(1):209-218, 1999.

[18] G. Dattoli, P. Ottaviani, A. Torre, and L. Vázquez. Evolution operator equations: integration with algebraic and finite difference methods. applications to physical problems in classical and quantum mechanics and quantum field theory. La Rivista del Nuovo Cimento (1978-1999), 20(2):3-133, 1997.

[19] G. Dattoli, P. E. Ricci, and C. Cesarano. A note on legendre polynomials. International Journal of Nonlinear Sciences and Numerical Simulation, 2(4):365-370, 2001.

[20] G. Dattoli and A. Torre. Operational methods and two variable laguerre polynomials. Atti Acc. Sc. Torino, 132:1-7, 1998. 
[21] G. Dattoli, A. Torre, and S. Lorenzutta. Operational identities and properties of ordinary and generalized special functions. Journal of mathematical analysis and applications, 236(2):399-414, 1999.

[22] H. W. Gould, A. Hopper, et al. Operational formulas connected with two generalizations of hermite polynomials. Duke Mathematical Journal, 29(1):51-63, 1962.

[23] C. Jordan. Calculus of Finite Differences. Third Ed., Chelsea, Bronx, New York, 1965.

[24] H. L. Krall and O. Frink. A new class of orthogonal polynomials: The bessel polynomials. Transactions of the American Mathematical Society, 65(1):100-115, 1949.

[25] M. Lahiri. On a generalization of Hermite polynomials. Proc. Amer. Math. Soc., 27:117121, 1971.

[26] E. D. Rainville. Special functions. Reprint of 1960 first edition. Chelsea Publishig Co., Bronx, New York, 1971.

[27] S. Roman. The Umbral Calculus. Academic Press, New York, 1984.

[28] J. Steffensen. The poweroid, an extension of the mathematical notion of power. Acta Mathematica, 73(1):333-366, 1941.

[29] W. Wang. A determinantal approach to sheffer sequences. Linear Algebra and its Applications, 463:228-254, 2014.

[30] G. Yasmin. Some properties of Legendre-Gould Hopper polynomials and operational methods. Journal of Mathematical Analysis and Applications, 413(1):84-99, 2014. 